\title{
Chœurs de fondeurs :
}

Interpellations créatives et mises en mémoire

Chours de fondeurs : practice creative calling outs and remembering processes

Judith Hayem

\section{(2) OpenEdition \\ Journals}

Édition électronique

URL : http://journals.openedition.org/travailemploi/6245

DOI : 10.4000/travailemploi.6245

ISSN : 1775-416X

Éditeur

DARES - Ministère du Travail

Édition imprimée

Date de publication : 15 mars 2014

Pagination : 105-122

ISSN : 0224-4365

Référence électronique

Judith Hayem, « Chœurs de fondeurs : », Travail et Emploi [En ligne], 137 | janvier-mars 2014, mis en ligne le 01 janvier 2016, consulté le 02 mai 2019. URL : http://journals.openedition.org/

travailemploi/6245; DOI : 10.4000/travailemploi.6245 


\title{
Chœurs de fondeurs : interpellations créatives et mises en mémoire $\left.{ }^{*}\right)$
}

\author{
Judith Hayem ${ }^{(* *)}$
}

L'article examine comment, après s'être mobilisés contre la fermeture brutale de leur usine - qui eut lieu sans plan social ni dépollution du site - les ex-salariés de Metaleurop ont continué à la contester au sein de l'association Chours de fondeurs. Issue d'une intersyndicale, l'association fut créée immédiatement après la fermeture. Elle fut ralliée d'emblée et de manière durable par la quasitotalité des 830 salariés. Certains de ses membres ont entrepris de rétablir la parole des ouvriers sur ce qui avait eu lieu et de questionner, mettre en garde et proposer des alternatives aux délocalisations, en s'appuyant sur des créations artistiques. S'inspirant de la notion de "partage du sensible» chez RANCIĖRE, l'auteure qualifie cette pratique d'interpellations créatives et montre qu'elle est soutenue par une subjectivité égalitaire mais séquentielle. L'enquête sur la longue durée (2005-2010) montre en effet que les interpellations s'interrompent avec le retour des rapports hiérarchiques au sein de l'association qui se tourne alors vers des pratiques mémorielles plus classiques.

Il y a dix ans, en 2003, l'usine métallurgique de Metaleurop à Noyelles-Godault, à 30 kilomètres de Lille, établie depuis 1894 dans le Pas-de-Calais sous divers patronymes (Malfidano, Penarroya), et premier producteur de zinc et de plomb d'Europe, fut présentée comme l'une des premières usines rentables à être fermée et délocalisée dans des pays à bas coût de main-d'œuvre par un actionnaire américain ne respectant pas le droit français ${ }^{(1)}$. Insaisissable, la direction (GAULIN, 2005) laissa salariés et représentants de l'État sans vis-à-vis pour négocier. Comme le montrèrent par la suite plusieurs enquêtes menées par des journalistes et des réalisateurs (voir encadré 3, pp. 110-111), aidés par les salariés et leurs avocats ${ }^{(2)}$, Glencore, principal actionnaire, avait organisé depuis plusieurs années le démantèlement progressif de l'usine et sa mise en faillite frauduleuse afin, le moment venu, de se dédouaner derrière un montage de sociétés, de toute

$\left(^{*}\right)$ Merci à tous les Chœurs de fondeurs et tout particulièrement au «trio infernal» qui se reconnaîtra.

(**) Université Lille-1, Clersé (Centre lillois d'études et de recherches sociologiques et économiques); judith.hayem@ univ-lille1.fr

(1) En 2002, les employés de Daewoo subissaient le même sort en Lorraine. Cet événement a d'ailleurs aussi fait l'objet d'un travail artistique avec les salariés (Bon, 2004). Les processus de délocalisation n'étaient cependant pas une nouveauté dans d'autres secteurs, voir par exemple pour l'industrie textile (Surubaru, 2010).

(2) Plusieurs cadres de Metaleurop ont mené leur propre enquête sur les malversations de Glencore, maison mère de Metaleurop, et ont transmis des informations aux journalistes, ce qui leur a valu pressions et menaces mais a permis à ces derniers de faire éclater l'affaire ( $c f$. par exemple La Tribune, "Comment Glencore a fait main basse sur Metaleurop», 30 janvier 2003; Le Monde, "Comment Glencore a peu à peu dépecé Metaleurop », 1 ${ }^{\text {er }}$ mars 2003 ou encore Les Echos, «Derrière Metaleurop, un actionnaire très discret, Glencore, le trader devenu industriel», 18 février 2003). responsabilité relative à la fermeture du site. Olivier MAZADE (2013a) a notamment étudié l'incapacité des cadres de l'entreprise, confrontés pour certains à la surdité de leurs interlocuteurs, frappés pour d'autres d'une forme de déni de réalité, à prendre acte et dénoncer en temps réel le démantèlement progressif de Metaleurop. En janvier 2003, Metaleurop SA, annonça ainsi, par la voix de cet actionnaire principal, qu'elle ne prendrait en charge ni les frais de licenciement des 830 salariés, ni les dépenses de dépollution du site, particulièrement lourdes dans ce type d'industrie, pour ne pas compromettre sa stabilité financière.

Le retentissement médiatique de l'affaire, tant à l'échelon local que national, fut puissant et de longue durée. La presse nationale commenta l'événement pendant de longues semaines et le scandale écologique (ZuindEAU, LETOMBE, 2010), ajouté au désastre économique local, provoqua immédiatement de nombreuses réactions politiques - notamment des Verts, très critiques sur le premier aspect, et du gouvernement, de la Région et du Fonds social européen, sur le second. Ces derniers se substituèrent pour partie à l'employeur dans la gestion de l'aprèsfermeture, en particulier pour l'aide au reclassement des salariés. C'est en parlant de Metaleurop que Roselyne Bachelot, alors ministre déléguée à l'Ecologie, puis Jacques Chirac, président de la République, utilisèrent avec un fort retentissement médiatique le terme de «patrons voyous ${ }^{(3)}$ » qui a fait florès depuis.

(3) «Interview de $M^{\text {me }}$ Roselyne Bachelot-Narquin, ministre de l'Écologie et du Développement durable, à Europe 1 le 23 janvier 2003, sur la faillite de l'entreprise Metaleurop, la pollution du site par le plomb et sur la pollution due au naufrage du pétrolier Prestige», disponible en ligne à l'adresse : http://discours.vie-publique.fr/notices/033000310.html ; consulté le 8 avril 2014 et MAZADE (2013b). 


\section{Encadré 1}

\section{Actions mises en œuvre par Chœurs de fondeurs}

- Une longue bataille juridique pour faire reconnaître le caractère illégal du désengagement de l'employeur Metaleurop vis-à-vis de ses employés, le «lâchage » comme l'appellent les intéressés (voir encadré 2), et obtenir des dommages et intérêts.

- Un engagement pour la redynamisation économique du territoire(1) : participation à l'audit des entreprises volontaires pour la reprise du site, accompagnement à la création d'entreprises dans la région par d'anciens salariés (action étudiée par CoRTEEL, 2009a et 2009b).

- Un travail dit «d'accompagnement social» qui s'est traduit par l'appui de Chœurs de fondeurs à la cellule de reclassement de l'ANPE (Agence nationale pour l'emploi, devenue Pôle Emploi) destinée à favoriser le retour à l'emploi des anciens fondeurs (travail détaillé dans MAZADE, 2005 et 2010).

- Une lutte pour la reconnaissance des maladies professionnelles, notamment celles liées à l'amiante, aboutissant là-encore à un contentieux juridique (voir encadré 2).

- Et enfin, plus inattendue et originale, une série d'interpellations que je qualifie de «créatives " et qui cheminent au côté de ces différentes activités.

(1) Cette initiative, postérieure à la fermeture ici, n'est pas sans rappeler l'investissement des salariés belges de Cockerill - Arcelor Mittal lors de la progressive fermeture de l'usine métallurgique de l'autre côté de la frontière, investissement qui a été décrit par LOMBA (2013).

En dépit des conséquences graves sur le plan professionnel, personnel, familial et financier (Linhart et al., 2002; MAZADE, 2010), de leur abandon par l'entreprise dans une région déjà lourdement touchée par le chômage ${ }^{(4)}$, les anciens salariés se sont battus pendant près de dix ans pour faire reconnaître «le préjudice moral » qu'ils avaient subi et démontrer que l'usine aurait pu vivre et que la fermeture n'était pas une fatalité.

Après une première phase de mobilisation, organisée au travers d'une intersyndicale (CGT, FO, CFTC, CFDT, CFE-CGC ${ }^{(5)}$ ) pour tenter d'empêcher la fermeture de l'usine (deux mois et demi d'assemblées générales, de manifestations, d'adresses aux pouvoirs publics, d'interventions dans les médias, etc.), les salariés en lutte - ouvriers, personnels administratifs, contremaîtres et ingénieurs ${ }^{(6)}-$ se sont constitués, dès la signature du plan social, en une association baptisée Chœurs de fondeurs(7). Son ambition explicite était de pouvoir continuer le combat pour contester les licenciements et affirmer

(4) Dans le Pas-de-Calais, selon l'Insee, le chômage concerne $12,4 \%$ de la population en 2003 (contre $9,8 \%$ à l'échelle nationale), conséquence de la délocalisation de l'industrie textile dans les années 1960 et des fermetures successives de mines dans les années 1970. La fermeture de Metaleurop impacte aussi de nombreux sous-traitants locaux dépendant de son activité.

(5) Confédération générale du travail; Force ouvrière; Confédération française des travailleurs chrétiens ; Confédération française démocratique du travail; Confédération française de l'encadrement - Confédération générale des cadres.

(6) «En 2002, l'usine compte 830 salariés au total dont 40 cadres et ingénieurs $[4,8 \%], 333$ employés, techniciens et agents de maîtrise [40,2\%], 457 ouvriers [55\%] (dont 316 dans les fonderies de plomb et de zinc)» (MAZADE, 2010, pp. 28-29). (7) Les statuts de l'association établis le 10 avril 2003 stipulent que «chaque organisation syndicale représentée au CE de Metaleurop Nord peut proposer un membre, au moins 6 jours ouvrables avant l'Assemblée générale de création : la délégation obtenue organise le scrutin pour désignation des autres membres »; statuts disponibles en ligne à l'adresse : http://www. choeursdefondeurs.fr/56040650, consultés le 2 février 2014. l'importance de l'usine pour ceux qui y travaillaient et pour son territoire d'implantation. Et ce, au-delà de la fermeture définitive de celle-ci. Cette réorganisation inventive a permis aux ex-salariés de sortir du cadre défensif de la première phase de lutte contre les licenciements et la fermeture du site (CORTEEL et al., 2009) pour poursuivre la mobilisation sous des formes renouvelées et diversifiées (voir encadré 1).

Les quatre premières tâches présentées dans l'encadré 1 sont explicitement désignées comme buts de l'association dans ses statuts et sont organisées par des «commissions» en son sein. C'est sur la dernière, les interpellations créatives, qui sont absentes des statuts mais vont occuper une place importante dans son activité, que l'article se concentre: des œuvres littéraires, cinématographiques, musicales, créées par des ex-salariés eux-mêmes, avec ou par des artistes de métier ont rencontré un large écho et ont été débattues grâce à l'association; elles visaient à faire connaître la lutte des Metaleurop et, selon leurs propres termes, faire "entendre la voix des ouvriers». J'appelle ici interpellation créative l'usage fait par les Chœurs de fondeurs de ces œuvres, non pas seulement pour «recouvrer une intégrité» (BERGERON, DoraY, 2005), dénoncer une situation ou conserver une mémoire, mais pour dire soi-même la place qu'on occupe ou celle qu'on aimerait occuper et qui n'est pas celle qu'assignent les acteurs des champs médiatiques, politiques, économiques, détenteurs du pouvoir de parler sur et pour les classes populaires (BOURDIEU, 1982). Les ex-salariés qui pratiquent l'interpellation créative ne peuvent en effet prétendre constituer une organisation ou un processus politique à proprement parler; cependant leurs initiatives ont bien pour objectif de transformer le champ des possibles et en ce sens, elles touchent à la politique. Ces réflexions s'inspirent des propositions de Jacques RANCIÈRE $(2000,2007)$ sur la notion de «dissensus» dans le domaine artistique. Le philosophe démontre en effet que la création peut susciter des "scènes nouvelles», génératrices d'un questionnement politique. 


\section{Encadré 2 \\ Metaleurop, une bataille juridique de longue haleine}

L'affaire Metaleurop a fait l'objet de deux procès distincts étudiés par Olivier MAZADE (2013b) : le premier devant les tribunaux, le second devant les prud'hommes. Ils sont retracés dans le documentaire de John Paul Lepers, Jean-Michel Meurice et le DVD, Les Naufrageurs démasqués, réalisé par Attac-Romans et Chœurs de fondeurs.

La question de la responsabilité sociale de l'entreprise à l'égard d'une de ses filiales, enjeu du premier procès, a fait l'objet de plusieurs décisions successives et contradictoires. Après que le 16 décembre 2004, «la cour d'appel de Douai [ait étendu] la liquidation de Metaleurop Nord au groupe Metaleurop SA [de sorte que] sa responsabilité dans la faillite industrielle de la fonderie de Noyelles-Godault est établie [...], le 19 avril 2005, la Cour de cassation (Paris) annule l'extension de la liquidation judiciaire de Metaleurop Nord à sa maison mère estimant que la cour d'appel de Douai "s'était déterminée sur des motifs impropres pour [d'une part] caractériser la confusion de patrimoines entre Metaleurop Nord et Metaleurop SA" et, d'autre part, estimer que les deux sociétés entretenaient "des relations financières anormales constitutives d'une confusion du patrimoine" " (Cass. chambre commerciale, 19 avril 2005, n 05-10094). L'opération de «restructuration artificielle», attaquée devant le tribunal de grande instance de Béthune, a par ailleurs fait l'objet d'une condamnation par l'AMF (Autorité des marchés financiers) le 18 mai 2005.

Début 2006, l'association Chœurs de fondeurs entame un second procès et dépose une procédure devant le conseil des prud'hommes de Lens contre Metaleurop SA pour «licenciement abusif sans cause réelle». Cette fois Recylex (1) est condamnée en 2008 pour «licenciement sans cause réelle ni sérieuse» et doit verser 50000 euros à environ 600 ex-salariés. Ce jugement est confirmé en appel par la cour d'appel de Douai pour 30000 euros en décembre 2009. Le groupe Recylex pose un recours en cassation : son pourvoi est rejeté le 28 septembre 2011, la chambre sociale confirmant le jugement (Cass. soc., 28 septembre 2011, nº 10-12278).

Un troisième procès s'est ouvert très récemment : en 2013, 299 ex-salariés de Metaleurop attaquent leur employeur aux prud'hommes pour préjudice d'anxiété lié à leur exposition à l'amiante et au bouleversement de leurs conditions d'existence, avec des demandes approchant les 10 millions d'euros. Recyclex se défend par un communiqué de presse dans lequel il fait état de sa situation difficile suite aux procès successifs qui lui sont intentés (2). En l'absence de conciliation entre les parties lors des rencontres précédentes, la décision sur le préjudice d'anxiété est toujours pendante à ce jour.

Le calendrier des procédures judiciaires concernant Metaleurop Nord est disponible sur http://www.recylex.fr/ fr,actualites, calendrier-procedures-judiciaires.html (page consultée le 24 mars 2014).

(1) Recyclex est le nom qu'a pris l'entreprise après la fermeture de Metaleurop Nord.

(2) Communiqué disponible en ligne à l'adresse : www.recylex.fr/download.php ?fileid =871; consulté le 24 mars 2014.

Si je choisis cette référence plutôt que les catégories habituelles de la sociologie ou des sciences politiques dans l'examen de l'usage de la création, qui est loin de se limiter à cette seule mobilisation (BALASINSKI, MATHIEU, 2006; SinIGAGLiA, 2012), c'est en raison de son adéquation à la posture ${ }^{(8)}$ des salariés de Metaleurop. RANCIÈRE (2000, 2007) évoque l'efficacité du «dissensus» pour désigner «non pas le conflit des idées ou des sentiments» mais le «conflit de plusieurs régimes de sensorialité». Il ajoute que c'est par là que l'art, dans le régime de la séparation esthétique, se trouve toucher à ce qu'il nomme «la politique» soit, ce qui «rompt l'évidence sensible de l'ordre "naturel" qui destine les individus et les groupes au commandement ou à l'obéissance, à la vie publique ou à la vie privée, en les assignant d'abord à tel type d'espace ou de temps, à telle manière d'être, de voir et de dire» (RANCIÈRE, 2000, p. 66).

En l'occurrence, les Chœurs de fondeurs dénoncent le sort qui est le leur et militent aussi pour que soient

(8) Nous étudierons principalement ici la subjectivité des ex-salariés et secondairement seulement, les choix artistiques et militants des artistes comme l'a fait Audrey MarietTe (2005) à propos des films sur les fermetures. Les parcours des réalisateurs et des artistes qui ont travaillé sur Metaleurop présentent d'ailleurs certaines similarités avec les réalisateurs engagés contre le néolibéralisme qu'elle a étudiés. trouvés les moyens d'empêcher les délocalisations brutales, non seulement dans leur cas mais aussi dans d'autres. Surtout, ils affirment qu'il faut reconnaître l'existence des ouvriers, de l'usine et du travail, les spécificités et les qualités de celui-ci, ses apports et son importance pour l'industrie locale et nationale, et refusent le verdict économique de la fermeture imposée. Ils entreprennent ainsi de réinstaller de manière positive la question des ouvriers et du travail industriel dans le débat national et même, au-delà des frontières. J'examinerai cette volonté et interrogerai sa spécificité au regard des luttes qui l'ont précédée et de la conjoncture des années 2000. Comment les Chœurs de fondeurs qui pratiquent l'interpellation créative se représentent-ils leurs actes, leur impact, leurs enjeux ? Et pourquoi interrompent-ils ces créations fin 2007 alors que l'association, elle, perdure ? Avec une remarquable longévité, Chœurs de fondeurs a tenu sa dixième assemblée générale le 9 avril $2013^{\left({ }^{(9)}\right.}$, devant un parterre toujours dense mais dans une ambiance sensiblement distincte des premières années.

Cet article se base sur l'examen diachronique des activités et du devenir de l'association mené sur

(9) «Dix ans après, Chœurs de fondeurs continue de battre le fer», La voix du Nord, 9 avril 2013. 
la période de cinq ans (2005-2010) qu'a duré mon enquête. Il s'appuie sur vingt-trois entretiens recueillis par l'écrivain Frédéric FAJARDIE (2003) juste après l'annonce de la fermeture de l'usine ainsi que sur divers travaux consacrés à l'affaire Metaleurop et à l'association. Je mobilise également quinze entretiens réalisés par mes soins auprès des acteurs des mobilisations créatives, dix autres recueillis par CORTEEL et par DuHIN (2009) (10) ainsi que l'analyse des créations produites et de nombreuses observations effectuées lors des différentes assemblées générales $(\mathrm{AG})$ de l'association et à l'occasion d'interpellations et de sessions de travail dans les locaux de l'association ${ }^{(11)}$.

\section{Une association originale pour une lutte de longue haleine}

\section{Continuer "tous ensemble"}

Après la liquidation de Metaleurop qui ôte à l'intersyndicale toute représentativité, Chœurs de fondeurs se constitue comme un outil de combat afin notamment de porter la requête de ses membres devant les tribunaux - c'est là, les "piliers de l'association» ne s'y trompent pas, une motivation centrale pour y adhérer ${ }^{(12)}$. L'association (loi 1901) est lancée en avril 2003 dès qu'il devient évident que la fermeture du site est inéluctable; elle se définit comme «une association ajustée au sort des anciens Metaleurop $[\ldots] »$ vouée à «[les] identifier comme [une] entité par rapport aux communes pour obtenir des locaux et des subventions afin d'innover, de fonctionner»». L'adhésion est massive (600 adhérents dès la création puis rapidement $90 \%$ des 830 ex-salariés ${ }^{(13)}$ ) d'autant que Chœurs de fondeurs nait dans la continuité de la lutte, sans laisser aux salariés le temps de se laisser (trop) gagner par l'amertume d'un premier revers. Ils sont seuls à pouvoir adhérer, moyennant une cotisation modeste de dix euros annuels. Tous ne sont pas des membres actifs de l'association. «Ceux qui font

(10) Après un travail collectif dans le cadre d'un Bonus qualité recherche $(\mathrm{BQR})$ de l'université Paris-8, qui a donné lieu à un premier article, les trois chercheuses initialement réunies pour le projet ont chacune approfondi l'un des aspects de la mobilisation mais se sont communiqué les matériaux qu'elles avaient recueillis. Plusieurs entretiens ont été réalisés avec les mêmes interlocuteurs au fil du temps. Contrairement à FAJARDIE, et pour respecter l'anonymat auquel je me suis engagée auprès de mes interlocuteurs, les prénoms utilisés sont des pseudonymes. (11) Ce travail a fait l'objet d'une contribution, sur laquelle je m'appuie et dont j'utilise des extraits à plusieurs reprises dans cet article, au programme de recherche financé par le Puca (Plan urbanisme construction architecture) et dirigé par Bernard Eme, professeur à Lille-1, sur «La citoyenneté urbaine : formes d'engagement et enjeux de solidarité» sous le titre «La mobilisation créative des ex-ouvriers de Metaleurop Nord».

(12) Ce paragraphe se fonde sur des notes de terrain recueillies le 18 octobre 2006 par CORTEEL, DuHIN et moi-même et réunies dans un document unique. Propos entre guillemets tenus par Richard, ancien ingénieur et membre du CA de l'association.

(13) L'adhésion à l'association est restée stable au fil du temps. Il faudra donc attendre l'assemblée générale de 2014 pour juger si l'extinction des poursuites juridiques se traduit par une désaffection des membres. confiance» à Chœurs de fondeurs, et lui délèguent notamment la bataille devant les tribunaux, se distinguent de ceux qui vont parfois jusqu'à consacrer tout leur temps libre à l'association (voir tableau en annexe). Tous les salariés licenciés ne s'étaient pas non plus mobilisés contre la fermeture. Les plus engagés évaluent à «250-300» au moins le nombre stable de «ceux qui luttent» et sont de toutes les manifestations, présents à toutes les audiences au tribunal ${ }^{(14)}$.

Dans les entretiens, c'est cette dichotomie entre «ceux qui luttent» et «ceux qui ne luttent pas» qui exprime le choix de l'engagement. Pour les ex-salariés, s'engager dans la lutte est une question de «dignité», à recouvrir et à affirmer, qui fait d'eux des membres de ce qu'ils nomment «la classe ouvrière». Cette affirmation mérite qu'on s'y arrête. En effet, si la question de la dignité est récurrente dans toutes les luttes contre les fermetures de site, la notion de "classe ouvrière» en tant que telle est de moins en moins employée par les protagonistes. Elle réapparaît ici pour incarner une nouvelle figure ouvrière dans une période qu'avec Sylvain LAZARUS (2001) je qualifierais pourtant de postclassiste. Elle exprime la subjectivité particulière des Metaleurop dans une acception originale du terme. L'expression, appliquée à la situation considérée, évoque de manière conjointe chez un certain nombre de salariés leur passé d'ouvrier ${ }^{(15)}$, leur grand-père mineur, leur milieu d'origine populaire ou encore l'histoire sociale de la région. Elle ne se réfère pas principalement à un statut, un métier, un salaire, ou à d'autres conditions objectives de travail et de vie, puisque certains sont contremaîtres, comptables, voire ingénieurs. Elle désigne d'abord, pour tous ceux qui l'emploient, le choix de lutter ensemble et de refuser le sort prescrit par d'autres, contre «l'indignité» du renoncement à la lutte. C'est là la subjectivité commune, la décision qui fonde le collectif autant que l'antagonisme avec un patron ou des actionnaires. En effet, dans l'opposition à la fermeture, dans la volonté de proposer un autre possible, les ex-salariés mobilisés se considèrent comme des égaux, membres de la «classe ouvrière» et ce, quels que soient leurs statuts professionnels et hiérarchiques à l'usine et leurs origines sociales. En témoignent ces propos sur l'unité recueillis par FAJARDIE pendant la première phase de la mobilisation au début 2003 :

«Ce qu'il faut comprendre, c'est que, c'est pas une division ouvriers, maîtrises et cadres, la division c'était entre ceux qui luttent et les autres. Quand vous militez, le gars d'à-côté, c'est plus un fondeur ou un ingénieur, c'est un gars qui lutte avec vous. "

(Entretien recueilli par FAJARDIE avec Jean-Claude, 52 ans, ancien comptable [FAJARDIE, 2003, p. 35].)

(14) Dans ses notes de terrain, DuHin indique par exemple qu'une centaine de personnes est présente lors des audiences aux prud'hommes du 23 mars 2007 et du 8 juin 2007.

(15) Beaucoup sont entrés à l'usine à des postes peu qualifiés et ont fait l'expérience d'une importante promotion professionnelle en interne. 
«Durant la lutte, la maîtrise a été très responsable, absolument solidaire. C'est pareil pour les cadres, vraiment sur les positions des ouvriers. C'est pour ça que notre histoire est exceptionnelle. Ça, et le côté improvisé, je veux dire que les ordres n'arrivaient pas d'en haut, on discutait tout, on prenait les décisions entre nous, sans aucune hiérarchie d'aucune sorte. »

(Entretien recueilli par FAJARDIE avec Gérard, 54 ans, agent de maîtrise au laboratoire de contrôle et d'analyse [FAJARDIE, 2003, p. 35].)

Selon eux, la lutte aurait aboli les anciennes différences de statut hiérarchiques et professionnelles et rendu légitime tout un chacun à prendre la parole. De sorte que le «tous ensemble», traditionnellement scandé dans les manifestations depuis les mobilisations contre les retraites de 1995 et repris par les Metaleurop martèle l'unité mais aussi l'égalité de place et de poids au sein du collectif militant. "On est tous ensemble [...]. Y'a pas de cadre, pas d'ouvrier, c'est ça qui fait notre force ${ }^{(16)} 》$, résume Suzy, ancienne représentante de la CFE-CGC. Cet état d'esprit s'explique par le poids de la CGT dans l'usine, dont le discours unitaire sur les ingénieurs solidaires avec les ouvriers porté par l'UGICT (Union générale des ingénieurs, cadres et techniciens de la CGT), paraît mis en acte. Il doit aussi beaucoup à Farid, chef de file incontesté de l'intersyndicale. Fils d'un mineur marocain, ancien leader de la mobilisation étudiante contre les lois Devaquet, Farid est âgé de 36 ans au moment de la fermeture. Agent de maîtrise à l'atelier fusion depuis 1993 après une embauche à Metaleurop en 1991, Farid est devenu délégué syndical CGT en 1994, et assume lui-même la déconnexion potentielle entre son statut professionnel et son engagement syndical à la CGT :

"J'ai adhéré à la CGT parce que j'avais ces idées-là, [...] j'ai pas ressenti de problèmes entre mon appartenance à la classe ouvrière et mes études; et pas de gêne non plus, quand je suis devenu agent de maîtrise, même si les agents de maîtrise adhèrent presque automatiquement à la CGC.»

(Entretien recueilli par FAJARDIE avec Farid, 36 ans, agent de maîtrise à l'atelier plomb, employé comme agent de maîtrise chez Sita [FAJARDIE, 2003, pp. 125-132].)

Alors que les anciens délégués de la CGT avaient mauvaise presse auprès de nombreux salariés - qu'ils soient cadres ou ouvriers -, qui évoquent souvent leur corruption, leurs luttes d'influence au sein de l'usine, leur racisme latent, voire leur proximité avec le Front national (17), Farid incarne le renouveau et l'unité entre différents niveaux hiérarchiques, entre générations, et ne fait pas l'objet des soupçons qui flottaient sur ses

(16) Entretien recueilli par CORTEEL le 18 octobre 2006 avec Suzy, 52 ans, ancienne comptable, salariée en contrat à durée déterminée (CDD) par le biais du Fonds social européen (FSE) pour le suivi de la cellule de reclassement de l'ANPE.

(17) Metaleurop Nord est située à quelques kilomètres d'HéninBeaumont où le Front national a remporté les élections législatives de 2013 avec plus de 55\% des voix. Parmi la main-d'œuvre de l'usine, on trouve des ouvriers d'origine polonaise et maghrébine. prédécesseurs. L'adhésion à cette acception revisitée de l'unité de la classe ouvrière est cependant contestée par certains. À l'instar de Mario, agent de maîtrise, qui se souvient des différences de statut très marquées du temps de l'activité de l'usine. Selon lui, cette solidarité des cadres avec les ouvriers est conjoncturelle :

"Quand on a fait les postes pendant trente ans, on appartient vraiment à la classe ouvrière. [...] Je ne crois pas aux cadres qui se sentent d'un seul coup ouvriers quand leur cul sent le bûcher [...] [et] qu'on n'avait jamais vus dans les grèves d'avant et qui vous auraient marché dessus (18). "

(Entretien recueilli par FAJARDIE avec Mario, 52 ans, agent de maîtrise [FAJARDIE, 2003, pp. 61-67].)

Symétriquement, si l'association est présentée comme un lieu où les anciennes étiquettes doivent s'effacer pour permettre la poursuite du «tous ensemble», on verra que le poids respectif des différentes confédérations et des anciennes hiérarchies professionnelles y est encore latent et va se réaffirmer au fil du temps. Cependant, le principe d'égalité va être efficace pendant plusieurs années et connaître une mise en œuvre originale via le travail d'interpellations créatives.

\section{L'association et ses bénévoles}

Au local de l'association, initialement sis à la mairie de Noyelles-Godault, on trouve toujours trois ou quatre personnes, voire plus, occupées à mettre sous pli le bulletin semestriel de l'association, réunir les pièces manquantes pour le dossier des prud'hommes, saisir des cotes d'archives, etc. sans compter les visites plus ponctuelles des anciens salariés. Conformément à la composition salariale de l'usine (environ $6 \%$ de femmes), on trouve plus d'hommes que de femmes dans l'association. La moyenne d'âge des membres actifs tourne autour de 50 ans, un âge légèrement supérieur à celui de l'ensemble des salariés; une partie d'entre eux est dispensée progressivement de recherche d'emploi(19). Pour les plus jeunes, le retour à l'emploi ou le temps consacré à la quête de celui-ci a rapidement pris le pas sur les engagements associatifs. Quant aux ouvriers plus âgés et proches de la retraite au moment de la fermeture, ils ne sont assidus qu'aux AG ou lors des opérations mémorielles (expositions, commémorations). Les animateurs et dirigeants de l'association ont en commun d'avoir souvent été des «militants» avant la fermeture (voir tableau en annexe). On retrouve parmi cette trentaine d'individus des ex-délégués syndicaux ou des militants associatifs, qui inscrivent leur engagement actuel dans la continuité de leurs expériences passées.

Comme le révèle le tableau en annexe, les cadres et agents de maîtrise sont largement surreprésentés

(18) Dans le livre, Mario est le seul à tenir cette position.

(19) Au moment de la fermeture, la moyenne d'âge de l'ensemble des salariés est de 47 ans : $6,9 \%$ des effectifs à reclasser ont plus de 55 ans et s'approchent d'une mesure d'âge les exonérant de recherche d'emploi; $63,2 \%$ ont plus de 44 ans. Pour plus de détails voir (MAZADE, 2006, 2010). 
par rapport aux ouvriers et aux employées dans le conseil d'administration de l'association. Ils y assument notamment des tâches de comptabilité, de rédaction ou d'expertise en rapport avec leurs compétences passées. Au sein de cet ensemble plus vaste, les porteurs d'interpellation créative représentent un sous-groupe fluctuant au gré des initiatives. Le trio le plus actif et le plus improbable réunit trois hommes, Pierre, Vladimir et Louis, soit un ingénieur, un agent de maîtrise et un ouvrier conducteur de locomotive sur le site, qui ne se connaissaient pas avant la fermeture. Pierre a fait une partie de sa carrière à l'étranger et est passionné d'égyptologie quand Louis affecte à l'occasion de parler ch'ti pour bien marquer sa condition ouvrière et son ancrage local.

Les outils artistiques choisis ne relèvent pas du hasard. Il s'agit le plus souvent de passions et de pratiques anciennes qui ont trouvé, dans la mobilisation puis au sein de l'association, l'occasion de s'employer ou de se révéler: premières expériences d'écriture, salvatrice pour Vladimir, agent de maîtrise «traumatisé par la fermeture» (Knopik, 2004, voir encadré 3), ou enthousiasmante pour Pierre, ingénieur passionné d'histoire et d'archéologie; documentation photographique de la destruction de l'usine et des actions menées par l'association pour Louis, conducteur de train qui « a commencé la photo en même temps que sa première cigarette»; enregistrement d'un disque de chansons pour faire connaître la lutte par Timothée, électricien, musicien semi-professionnel; création de films au service du témoignage ou pour reconstituer l'histoire du site par Alain, vidéaste amateur; acteur inattendu du documentaire qui lui est consacré pour Michel, par exemple (voir tableau en annexe et encadré 3).

\section{Encadré 3}

\section{Cuvres relatives à Metaleurop}

\section{OEuvres réalisées par ou avec des professionnels}

\section{Films documentaires}

- Bruandel J., Michel G. (2004), Metaleurop : du chômage au braquage, film documentaire, 20 min., présenté par Benoît Duquesne pour l'émission de France 2, Complément d'enquête. Production : France 2.

- Czubeck S., Lallement G. (2004), Le conflit Metaleurop, film documentaire, $1 \mathrm{~h}$ 18. Coproduction : Flight Production. Avant-première le 11 septembre 2004 à Roubaix. Première diffusion télévisée : 15 janvier 2005 sur France 3, Nord-Pas-de-Calais.

- Czubeck S., Mournaud C. (2008), Les années de plomb, film documentaire, 52 min. Coproduction : CRRAV, France 3, Nord-Pas-de-Calais. Première diffusion : octobre 2008.

- Lepers J. P. (2003), Metaleurop : l'autre guerre..., film documentaire, 1 h. Coproduction : Canal +. Première diffusion télévisée : avril 2003 sur Canal +.

- Mazery P. (2004), Glencore : la multinationale des flibustiers de l'économie, film documentaire pour Lundi investigation, 39 min. Coproduction : Canal +. Première diffusion télévisée : 5 janvier 2004 sur Canal +.

- Meurice J.-M., Dauriac C. (2004), L'éléphant, la fourmi et l'État. Aujourd'hui Metaleurop, demain... ?, film documentaire, 1 h 30. Coproduction : Arte France, Cinétévé, Anthracite. Première diffusion télévisée : 2 février 2004 sur Arte.

- Venemmani J.-M. (2004), Metaleurop, Germinal 2003, film documentaire, 1 h. Coproduction : Phares \& Balises. Première diffusion télévisée : mars 2004 sur France 3.

\section{Livres}

- Betaucourt X., Loyer J.-L., Barroux S. (2006), Noir métal, au cœur de Metaleurop, Paris, Delcourt.

- Dessaint P. (2010), Les derniers jours d'un homme, Paris, Rivages. Fajardie F. (2003), Metaleurop : paroles ouvrières, Paris, Mille et une nuits.

\section{Photographies}

- Dhaluin C. (2003), Reportage photographique sur Metaleurop, consultable sur le site de divergence images : http://www.divergence-images.com/recherche/metaleurop\%20nord/; consulté le 24 mars 2014.

\section{CEuvres réalisées par des amateurs}

\section{Films documentaires}

- CDF, Attac-Romans (2004), Metaleurop : les naufrageurs démasqués, film documentaire, 50 min. Coproduction : CDF et Attac-Romans (diffusion Chœurs de fondeurs). Première diffusion : avant-première le 16 décembre 2004 après le délibéré du tribunal de Douai.

- Fondre en chœurs, Metaleurop... 3 ans après, film documentaire collectif des ex-Metaleurop, 60 min. Production : CDF. Première diffusion lors de l'exposition Fondre en chœurs en 2006. Déposé aux Archives du monde du travail de Roubaix.

- Puchband L. (2004), Metaleurop : les fondeurs se souviennent, film documentaire, 5 min. Production : CDF. 


\section{Livre}

- Fermetures de boîtes... et après ? (2007), Actes des rencontres à la Maison des hommes et des techniques à Nantes, mars 2005, Maison des hommes et des techniques, Édition du Centre d'histoire du travail, Nantes (livre + cédérom).

- Knopik S. (2004), Jour comme un autre, Lille, Éditions du Sansonnet.

\section{Photographies}

- Pruvost A. et D. (2006), Stanis, Jacques et les autres, Montage photos et sons d'une dizaine de minutes réalisé avec des photos de Jacques Wyart et le texte de Stanis Knopik lu par J.-C. Van Fluten, récompensé par le premier prix de la Ligue des droits de l'Homme.

- Wyart J., Metaleurop 2003-2006, CD, 171 photos.

- Wyart J., Conflit Metaleurop, 3 mois de lutte, CD, 830 photos.

\section{Disques de musique}

- Chevallier P. (2003), Métallo, CD 11 titres, Droit de cité, Mairie de Harne, Sing Studio.

- Marie-Lore, Monde ouvrier, CD 2 titres enregistré et écrit par Marie-Lore, propriétaire du café-restaurant de Noyelles-Godault, situé dans la rue principale.

\section{Les interpellations créatives}

Plus encore que la qualité intrinsèque des œuvres, qu'elles soient amateures ou professionnelles, c'est la façon dont certains Chœurs de fondeurs les ont nourries, présentées, débattues, commentées en de multiples occasions et devant des publics variés (lycéens, cinéphiles, magistrats, politiques, étudiants, ouvriers et/ou syndicalistes en lutte dans d'autres usines et parfois même dans d'autres pays, etc.) qui leur confère le statut d'interpellations créatives (voir encadré 4). L'ouvrage de FAJARDIE (2003) en est un exemple paradigmatique en regard duquel je présenterai ensuite les autres œuvres et leurs usages par les Chœurs de fondeurs.

\section{Le travail des "porteurs de parole"}

Metaleurop. Paroles ouvrières. Tel est le titre significatif donné par l'auteur de polars et de romans historiques, Frédéric FAJARDIE (2003) au livre d'entretiens qu'il a recueillis auprès des salariés licenciés de Metaleurop Nord début 2003 et dont l'intégralité des droits d'auteur a été reversée à l'association. Comme le soulignait un des membres de Chœurs de fondeurs, en rendant hommage à l'écrivain décédé en 2008, «en donnant la parole» aux gens, en s'intéressant «au sort des ex-Metaleurop, de leur vie, de leur lutte et de ce monde ouvrier si absent [des] médias et des préoccupations des élus ${ }^{(20)} 》$, et en créant l'opportunité pour cette parole d'être diffusée sous la forme d'un livre, FAJARDIE a offert des moyens financiers aux fondeurs ${ }^{(21)}$ mais il a surtout proposé un ton et une manière : la prise de parole directe et l'interpellation qui font apparaître et/ou perdurer de nouveaux enjeux dans le débat public.

(20) Extrait d'un courrier, communiqué à l'auteure et adressé à la veuve de FAJARDIE par Pierre, 61 ans, ancien ingénieur, dispensé de recherche d'emploi.

(21) Vendu 10 euros, le livre a permis à la caisse de l'association d'engranger d'emblée plusieurs milliers d'euros.
Sollicité par des lecteurs ouvriers pour «donner une forme à leur mémoire ${ }^{(22)} 》$, FAJARDIE a préféré consigner leurs idées et leur ressenti sur le licenciement que leur faire évoquer le passé. Dans la préface de l'ouvrage, il replace son choix dans la lignée de ses études de sociologie et plus encore, dans la suite de son passé de militant «maoïste» (Ross, 2005). Cette ancienne secrétaire l'a bien noté qui souligne, "je trouvais ça bien [...] qu'on puisse dévoiler le fond des personnes [...], de ce qu'ils pensent en fait, de ce que les gens pensent ${ }^{(23)}$ ». L'auteur ne s'est fait ni biographe, ni représentant des ouvriers au sens où ils lui auraient délégué le soin de parler pour eux et à leur place au titre de sa capacité d'écrivain, de son aura ou de sa légitimité. Au contraire, il offre à la parole ouvrière une modalité d'expression autonome et adopte un rôle de messager et non d'interprète. Il se fait non pas porte-parole mais "porteur de paroles", selon le titre même que lui confèrent les ex-salariés. «Ces paroles [...], ces voix qui se sont élevées toutes ensemble dès le lendemain [de la fermeture] et se firent entendre toutes ensemble sans interruption », rappelle l'éditorial du premier Écrit du Chøur ${ }^{(24)}$; "c'est le rôle des intellectuels de nous aider à les porter ${ }^{(25) »,}$ affirme explicitement un bénévole à l'occasion d'une rencontre avec des réalisateurs et l'écrivain. Ce vocabulaire (maoïste, rôle des intellectuels) et les prescriptions qui l'accompagnent dénotent une filiation avec les luttes des années 1970 (MATHIEU, 2009) mais on est ici face à des décisions indivi-

(22) L'auteur s'exprime en ces termes dans la préface du livre, p. 15 .

(23) Entretien recueilli par Judith Hayem le 27 novembre 2007 avec Marie, 55 ans, ancienne secrétaire, en recherche d'emploi.

(24) Écrit du Chœur. Journal de l'association des ex-salariés de Metaleurop, $\mathrm{n}^{\circ} 1$. Nul hasard non plus dans le choix de l'appellation du collectif qui a bien trait à la capacité de dire à l'unisson.

(25) Propos de Pierre, 59 ans, ancien ingénieur, recueillis par Judith Hayem lors de la rencontre organisée le 15 décembre 2005. 
duelles et non plus de collectifs organisés de sorte qu'on ne saurait parler d'une «personnalisation» de la lutte pour les artistes / intellectuels discrets qui s'intéressent à Chœurs de fondeurs (Mouchard, 2009), ni d'un «établissement» pour décrire la pratique de FaJARDIE. Moins que les artistes ce sont leurs œuvres en devenir et ce qu'elles pouvaient porter qui ont mobilisé les Chœurs de fondeurs avec la conviction que "même s'il s'agit de livres, de $\mathrm{CD}$, de cassettes, ce qui peut paraître "ludique" est en fait porteur de nos messages, ceci donne lieu à des contacts, initie des débats ${ }^{(26)}$ ».

Effectivement, les six films et documentaires consacrés à Metaleurop (voir l'encadré 3) ont souvent été réalisés en collaboration étroite avec les ex-salariés. D'une part, parce que ceux-ci en sont les acteurs directs ou indirects, lorsque Stéphane Czubeck les suit dans toutes les AG et toutes les manifestations, dès les premiers moments $\mathrm{du}$ conflit, ou quand Jean-Michel Vennemani plante, pendant plusieurs mois, sa caméra dans l'intimité d'une famille frappée par le licenciement, suivant heure par heure, sans jamais intervenir, ce qu'il advient de leur vie après la fermeture, selon le principe même de l'émission Strip Tease pour laquelle il tourne. Mais ils sont aussi les interlocuteurs directs des réalisateurs pendant le tournage des films. Ainsi, plutôt que d'avoir recours à une voix off, Jean-Michel Meurice choisit d'intercaler dans son film des plans fixes sur fond noir devant lesquels les salariés de l'usine s'expriment. Seul John Paul Lepers choisit, lui, d'aller interroger avec impertinence les responsables de la fermeture, traquant jusque dans le canton de Zug en Suisse les grands patrons de Glencore et les parlementaires décisionnaires, dans un style qui rappelle celui de ses débuts au Vrai Journal de Karl Zéro sur Canal +.

Certains films sont descriptifs et témoignent simplement de ce qui s'est passé (Metaleurop, Germinal 2003 ou Le conflit Metaleurop). D'autres sont des films ouvertement militants, construits comme des tracts ou des réquisitoires. Ils interrogent : à quand des lois prévenant les licenciements? À quel niveau prendre des mesures (national, européen, mondial) pour empêcher les délocalisations ? Beaucoup démontrent et expliquent ce qui a eu lieu, ce qui a rendu possible un tel «lâchage» de l'entreprise et mettent aussi en valeur la ténacité du combat des ex-salariés confrontés au licenciement. Et lorsque dans L'éléphant, la fourmi et l'État, Meurice déclare : "Avec ce film, nous souhaitons dénoncer les patrons voyous et interpeller nos politiques », on retrouve là l'un des leitmotive des entretiens recueillis. Ce n'est pas une coïncidence. En effet, une longue correspondance par mails entre le réalisateur et certains Chœurs de fondeurs,

(26) Écrit du Chœur. Journal de l'association des ex-salariés de Metaleurop, $\mathrm{n}^{\circ} 1$. consignée dans les archives de l'association, témoigne du caractère co-construit du film. Forts de leurs investigations juridiques sur les malversations de Glencore et Metaleurop, certains cadres et ingénieurs avaient pu guider Meurice ou Lepers vers les interlocuteurs pertinents pour leurs enquêtes en France ou en Suisse. Enfin, la coproduction est encore plus explicite dans le cas du documentaire rigoureux produit par Attac-Romans et les Chœurs de fondeurs pour "dégager [des] propositions qui doivent permettre d'introduire un débat ${ }^{(27)} \gg$.

Hormis FAJARDIE, la rencontre de ces artistes avec Metaleurop ne relève pas d'un engagement volontaire et au long cours aux côtés de l'association. Les réalisateurs ont probablement autant vu dans Metaleurop un «bon sujet», une «bonne occasion ${ }^{(28)}$ 》 qu'une cause à défendre. Cependant les échanges noués avec certains ex-salariés font que la plupart de leurs films dépassent le simple témoignage pour porter des thèses élaborées avec eux autant qu'à leur endroit. Les Chœurs de fondeurs ont bien cherché à faire d'eux des "porteurs de [leurs] paroles» et ce sont ces thèses dont ils vont explicitement se saisir pour poursuivre l'interpellation et les débats après la sortie des films.

A contrario, quand les initiatives artistiques sur Metaleurop se font sans leur appui, ou leur déplaisent, les Chœurs de fondeurs les boycottent parfois. La pile de bandes dessinées Noir metal (Betaucourt, Loyer, 2006, voir encadré 3) fraîchement publiées, a ainsi passé toute l'exposition Fondre en cheurs organisée par l'association en 2006 sous une table, sans être proposée à la vente après que les anciens salariés l'aient feuilletée. Cette "caricature de l'ouvrier nordiste [...] alcoolique et raciste» a déplu : «Je ne me suis pas reconnu dans l'histoire du mec qui arrive bourré au boulot qui picole dans les ateliers et [qui va pêcher] du poisson plombé», déplore un des interviewés, même s'il admet que "ça correspond p'têt à une réalité chez certaines personnes ${ }^{(29)} 》$. Pour le scénariste et le dessinateur, originaires de la région, et dont la famille a été touchée par des cancers consécutifs à la pollution industrielle, il y avait sans doute là une réalité bonne à dire. Mais elle n'était pas celle que les fondeurs voulaient promouvoir. Ce n'était pas l'image d'eux-mêmes qu'ils souhaitaient voir diffuser. Pas plus qu'ils n'ont apprécié le second opus de Czubeck sur le thème de la plombémie consécutive à la pollution causée par le site industriel dans lequel le réalisateur déploie la thèse d'une omerta : de peur de perdre leur emploi si l'usine fermait, les ex-salariés

(27) Jacquette du DVD, Metaleurop, les naufrageurs démasqués.

(28) Czubeck a commencé à filmer la lutte par hasard en faisant des repérages pour un sujet sur la pollution, objet de son second film Les années de plomb.

(29) Propos recueillis lors de l'exposition auprès de Louis, 55 ans, ancien ouvrier conducteur de locomotive dans l'entreprise, retraité. 
auraient préféré faire silence sur la pollution dont ils étaient victimes. Fait notable, si minutieusement documentées soient-elles, aucune de ces deux œuvres ne parle jamais de la lutte contre la fermeture, ni de la mobilisation des Chœurs de fondeurs pour la reconnaissance des maladies professionnelles, notamment celles liées à la pollution au plomb et à l'amiante. Si les ouvriers y sont interrogés comme tels, c'est en tant que victimes passives ou même volontaires de la pollution.

En s'appuyant sur d'autres œuvres pour leurs interpellations créatives, les ex-salariés ne cherchent pas à falsifier la réalité, ils prennent une position alternative dans le débat. Ils rejettent tout particulièrement le rôle de victime passive et/ou impuissante. L'un d'entre eux le fit savoir lors du débat houleux entre militants écologistes et militants communistes qui suivit la projection du film Les Années de plomb au Cabaret de l'union à Roubaix le 21 novembre 2005. Pierre, ancien ingénieur de Metaleurop, insista sur la lutte menée par les salariés contre la fermeture en mettant en avant l'impact social et pas seulement écologique de cette dernière sur le bassin d'emploi. Il signala au public que j'enquêtais sur les Chœurs de fondeurs, m'enjoignant à témoigner de la situation des ex-salariés. C'est bien là tout l'enjeu des interpellations créatives : ne pas

\section{Encadré 4}

\section{Interventions et interpellations créatives des Chœurs de fondeurs, une sélection}

Interventions radiophoniques des membres de CDF

- 15 janvier 2004, intervention dans l'émission Passé simple avec Myriam Schelscher et Cédric Faiche;

- 12 février 2004, intervention sur RFI dans l'émission Le monde change de Patrick Champré;

- 24 janvier 2005, intervention sur Radio libertaire;

- 4 juin 2005, participation à une émission sur France culture avec Olivier Mazade, Frédéric Fajardie, Stéphane Czubeck.

Déplacements des CDF, individuellement ou en groupe de 2 à 3 personnes pour accompagner et commenter des œuvres ou des événements

- octobre 2003, intervention au séminaire du syndicat de la magistrature, Forum social européen, Paris;

- octobre 2003, création d'un groupe de travail avec Attac-Romans dans la perspective de réaliser Les Naufrageurs démasqués, Romans (Rhône-Alpes);

- 18 octobre 2003, invitation à l'Ire en fête au Palais Saint-Waast dans le cadre de l'association Colères du présent, Arras (Pas-de-Calais);

- 11 mai 2004, projection d'un documentaire à l'initiative de l'association Envie d'agir au collège Paul-Langevin : échanges avec les élèves de troisième autour de l'histoire du bassin minier à Rouvroy (Pas-de-Calais);

- 15 mai - 15 juin 2004, exposition des photos de Metaleurop de Cédric Dhalluin au Transphotographique 2004, Lille (Nord);

- 1er juin 2004, intervention auprès d'étudiants de Master de l'université Lille-2, en parallèle de l'exposition des photos de Cédric Dhalluin, Lille;

- décembre 2004, participation aux Bobines sociales de Belleville, autour du film Metaleurop, Germinal 2003, Paris;

- janvier 2005, Projection de Metaleurop, Germinal 2003 et présentation des photos de Cédric Dhalluin, MJC, Saint-André (Nord);

- courant 2005, diffusion du film Le conflit Metaleurop, avec le soutien de la Ligue des droits de l'Homme, à Boulogne le 24 février 2005, Calais le 25, Lille le 28, Arras le 3 mars, Hénin-Beaumont le 16 mars, Campagne-lèsHesdin le 19 mars, Saint-Pol-sur-Ternoise le 25 mars, Lens le 7 octobre, Wingles le 18 octobre (Nord et Pas-deCalais);

- mars 2005, colloque Fermetures de boîtes... et après ?, Maison des hommes et des techniques, Nantes (LoireAtlantique);

- avril 2005, invitation aux journées de l'Afip (Association de formation et d'information pour le développement d'initiatives rurales), sur le thème "Nouvelles formes de mobilisation », Villeurbane (Rhône);

- 21 avril 2005, rencontre entre les anciens salariés et Stéphane Czubeck autour de son film, au Cabaret de I'union, en collaboration avec les Archives du monde du travail, Roubaix (Nord);

- 28 avril 2005, lectures du livre de Frédéric Fajardie à Heuringhem (Pas-de-Calais);

- décembre 2005, rencontre avec les étudiants et enseignants de la MST (maîtrise des sciences et techniques) Banlieues à l'université Paris-8 en présence de Jean-Michel Vennemani et Frédéric Fajardie, Saint-Denis (SeineSaint-Denis);

- 25-29 août 2005, participation des Chœurs de fondeurs et des Lustrucru (ex-salariés de l'usine de pâtes Lustucru d'Arles, Bouches-du-Rhône) à l'université du Mouvement national des chômeurs et précaires, Paris;

- 25 mars - 4 avril 2006, exposition Fondre en chœurs à Courcelles-les-Lens (Pas-de-Calais). L'exposition est très fréquentée, écoles et lycées de la région y affluent; des lycéens de Nantes font même le déplacement;

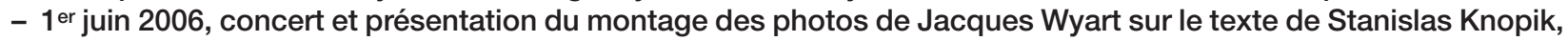
à Hénin-Beaumont (Pas-de-Calais);

- 2007, intervention à l'université de Reims auprès d'étudiants d'une formation en travail social, Reims (Marne); - janvier 2008, intervention devant les syndicats portugais à Coïmbra (Portugal). 
laisser confisquer de manière univoque, ni par des journalistes, ni par des militants politiques ou des analystes économiques, ni même par des artistes, la pluralité du possible, qu'il soit advenu (ce qui s'est vraiment joué à la fermeture de l'usine et ce qui aurait pourtant pu se jouer d'autre) ou encore à venir. Il est ici question d'une "réinvention de la prise de parole», explique un de mes interlocuteurs, ou encore, au sens même de RANCIÈRE, d'une «réappropriation des moyens de communication». Pour l'apprécier, examinons à présent l'usage que Chœurs de fondeurs a fait des films et autres créations (voir encadré 4) et surtout ce que les interviewés disent du sens de leur action.

\section{"Réinventer la prise de parole ouvrière"}

Pour la plupart des porteurs d'interpellations créatives, prendre la parole à la radio ou en public, se rendre à l'université ou dans un congrès syndical ou simplement prendre le train pour traverser la France à la rencontre de collègues en lutte comme ils le firent régulièrement entre 2003 et 2007, était impensable avant la fermeture. Interrogés sur le sens qu'ils attribuent à leurs créations et à la diffusion et au commentaire des œuvres créées, les Chœurs de fondeurs déclinent une série d'arguments que nous allons maintenant examiner.

\section{Une affirmation d'existence}

Janvier 2008, Coïmbra. Devant un parterre de syndicalistes portugais à l'invitation desquels il a répondu, Pierre ancien ingénieur à Metaleurop déclare ${ }^{(30)}$ :

\begin{abstract}
"Chaque fois, c'est avec une grande émotion de revoir aujourd'hui tous ces films documentaires. [...] Jean-Michel Meurice, dans un des plus fameux films documentaires sur l'affaire Metaleurop-Glencore, L'éléphant, la fourmi et l'État : aujourd'hui Metaleurop, demain... ?, posait la question des rapports entre l'État, le patron voyou - l'éléphant, Glencore - et les salariés de Metaleurop, les 830 fourmis. Et chaque fois aussi, c'est avec une grande émotion de reprendre la parole et de dire à tous ceux qui luttent: nous, les petites fourmis, nous sommes avec vous et redire à tous que la plus petite fourmi peut rendre fou le plus gros des éléphants.»
\end{abstract}

À l'instar, des propos rapportés ci-dessus, le premier enjeu de la prise de parole est une affirmation d'existence et de capacité d'action de la part de ceux qui s'expriment; et ce, quel que soit le rapport de forces initial. La prise de parole manifeste la volonté que ces existences ne soient pas niées mais reconnues. Tous les entretiens indiquent combien la fermeture soudaine qui s'est imposée à l'ensemble du personnel a été vécue comme un

(30) Pour l'occasion et afin de préparer la traduction, Pierre a rédigé son texte, dont il m'a remis une copie. triple déni d'existence. D'abord, parce qu'elle intervient si brutalement que les Metaleurop ont le sentiment d'être attaqués dans leur intégrité physique. Certains parlent de "tremblement de terre $^{(31)}$ » d'autres vont jusqu'à parler de «viol» pour décrire l'effet de cette fermeture sans «négociation, sans préparation, sans plan social, $d u$ jour au lendemain, clac (32)!», comme si aucune mesure n'était nécessaire car personne ne serait en réalité concerné par cette cessation d'activité. En second lieu, c'est sur le plan de leur identité que les Metaleurop se sentent niés. Non seulement ils perdent leur emploi et le statut qu'il leur confère mais la disparition physique de leur lieu de travail, dernier fleuron de la métallurgie en Nord-Pas-deCalais, qui fut au final entièrement rasé, les prive de tout repère objectif pour se définir aux yeux du monde et de leur famille et se remémorer ce qu'a été leur vie. L'image qu'ils veulent donner en contrepoint de cet effacement est celle des " ouvriers qui ont travaillé là-dedans, qui aiment ce qu'ils faisaient! [...] $80 \%$ des ouvriers [qui] ont travaillé dans cette usine-là [...] n'iraient pas ailleurs pour tout l'or du monde. C'était leur usine ${ }^{(33) ! ! ~ » ~ E n f i n, ~ t r o i s i e ̀ m e ~ a s p e c t ~ d u ~ d e ́ n i, ~ c e ~ q u i ~}$ est dit et présenté au public de la situation privilégie la problématique écologiste au point de leur donner le sentiment de ne pas avoir eux-mêmes leur place dans le tableau, comme si leur point de vue et leur sort étaient indifférents :

«On n'a pas lutté que contre les patrons voyous et les flics : contre nous on avait les Verts. [...] La télé, la presse, tout le monde reprenait ce que disaient les Verts sur la pollution [du site] : pour Glencore, pendant des mois ça a été du pain béni.»

(Entretien réalisé par Frédéric FAJARDIE, avec Ginette et Claude, comptables, mari et femme [FAJARDIE, 2003, p. 35].)

«Toujours le point de vue des Verts. Par contre, le point de vue des ouvriers qui ont des familles et tout... Pourtant on était plus exposés que tout le monde.»

(Entretien recueilli par Judith Hayem le 14 novembre 2007 avec Michel, 53 ans, ancien agent de maîtrise, créateur et directeur de la Scop Aced.)

En prenant la parole en leur propre nom et à leur propre endroit, les Metaleurop entendent manifester leur présence et leur importance et contrer des propos jugés «diffamatoires》 ou «avilissants ${ }^{(34)} 》$

(31) Entretien recueilli par Judith Hayem le 14 novembre 2007 avec Vladimir, 49 ans, ancien agent de maitrise, en recherche d'emploi.

(32) Entretien recueilli par Judith Hayem, le 26 novembre 2007 avec Michel, 53 ans, ancien agent de maîtrise, créateur et directeur de la Scop Aced.

(33) Entretien recueilli par Judith Hayem le 11 décembre 2006 avec Louis, 55 ans, ancien ouvrier conducteur de locomotive sur le site, retraité.

(34) Entretien recueilli par Judith Hayem le 26 novembre 2007 avec Vladimir, 49 ans, ancien agent de maitrise, en recherche d'emploi. 
de manière récurrente. Ils entendent restaurer leur "dignité» et celle de leur région atteintes par ce qu'ils perçoivent comme des mensonges et surtout comme une volonté de les faire disparaître, sinon physiquement, en tout cas intellectuellement comme enjeu et objet de ce qu'il y a à penser à propos de la fermeture de l'usine et aussi comme personnes capables de cette pensée :

«La dignité, c'est la lutte, ne pas couler, ne pas se laisser avilir. On a montré au Nord-Pas-de-Calais et à la France qu'on était là.»

(Entretien recueilli par Judith Hayem le 11 décembre 2006 avec Richard, 60 ans, ancien ingénieur, dispensé de recherche d'emploi.)

«Tout s'explique par cette prise de parole qu'on a voulue les uns et les autres par rapport à la presse et la volonté de prendre la parole. Cette volonté de prendre la parole, de reconnaître le témoignage des gens qui se sont vraiment sentis diffamés. "

(Entretien recueilli par Judith Hayem le 11 décembre 2006 avec Pierre, 60 ans, ancien ingénieur, dispensé de recherche d'emploi.)

Qui plus est, en l'absence d'interlocuteurs patronaux, prendre la parole est une manière de contrer ce que mon interlocuteur précédent appelle, à juste titre, «la violence du non-dialogue».

\section{"La médiatisation à l'envers "}

Le second enjeu de la prise de parole consiste à faire émerger «la vérité» sur la fermeture, son processus, son impact, ses effets sur les employés et sur le territoire. Pour ce faire, et dans la continuité du conflit social initial, les membres de l'association ont conscience qu'il faut occuper le terrain médiatique avec leur propre version des faits, ce que l'un d'entre eux appelle «la médiatisation à l'envers ${ }^{(35)}$ ». C'est là que les outils créatifs trouvent leur utilité. Pour démontrer les rouages de l'opération réalisée par Glencore d'abord. Mais les outils créatifs servent aussi à "montrer» les choses, les rendre visibles telles qu'elles sont vécues par les salariés. Ainsi, l'un d'entre eux, musicien, qui a produit le disque Métallo au moment du conflit, explique :

"J'ai fait un disque parce que j'étais bousculé; montrer la simple vie d'un ouvrier, le contexte général depuis 77 déjà, le chômage qui a empiré Metaleurop, le textile, la sidérurgie. [...] Faire parler de l'action des CDF, comme Allende les chansons pour le Chili. Laisser une trace, c'est la vérité, c'est pas égocentrique pour moi, c'est bien quand ça fait pleurer les collègues. »

(Entretien recueilli par Judith Hayem le 14 novembre 2007 avec Thimothée, ancien ouvrier électricien, retravaille dans l'automobile.)

(35) Propos recueillis lors de multiples conversations informelles avec Pierre, 60 ans, ancien ingénieur, dispensé de recherche d'emploi.
Tandis que Michel, héros de Metaleurop, Germinal 2003, se justifie face aux critiques éventuelles de voyeurisme et explique :

"Bon y'a des gens qui l'ont plus ou moins bien pris, Germinal 2003, certains m'ont dit : "c'est du cinéma", d'autres : "ça t'a aidé”. Mais pour moi, c'est tout du moins... laisser... faire montrer le mal que ça peut faire une fermeture d'usine sur... une famille hein [il est très ému et sa voix tremble], détruire des gens comme ça, simplement sur des...»

(Entretien recueilli par Judith Hayem le 26 novembre 2007 avec Michel, 53 ans, ancien agent de maîtrise, créateur et directeur de la Scop Aced.)

Il s'agit de rétablir le «drame humain (36)» qui se cache derrière les chiffres des licenciements et les informations factuelles. Incarner ce drame, en se mettant soi-même en scène est aussi une manière de faire exister ces ouvriers que l'on voudrait balayer. Notons cependant que si l'usage de l'émotion et de l'empathie est ici explicite, dans l'esprit de nos interlocuteurs, le propos des films et des livres évoqués n'est ni misérabiliste, ni nostalgique. Tout au contraire, parler du "drame» est une modalité de combat, d'où les propos du protagoniste de Metaleurop, Germinal 2003 :

"Il faut redresser la tête et même quand on est au fond du trou, y'a toujours un espoir. Parce que, quand on est au fond du trou, y'a celui qui dit, ben soit j'me fous en l'air, soit il se fout dans l'alcool et puis il se détruit quand même, soit il prend son courage à deux mains et puis bon... C'est de se dire, rien n'est jamais fini (37). "

(Entretien recueilli par Judith Hayem le 14 novembre 2007 avec Michel, 53 ans, ancien agent de maîtrise, créateur et directeur de la Scop Aced.)

De même, l'auteur du petit livre Jour comme un autre explique la nécessité de "parler $d u$ drame» parce qu'il "fallait que ce soit une mise en garde ${ }^{(38)} 》$. Il décrit une rage de dénoncer dans laquelle «les films, la manif [sont] du même ordre d'action. Une arme pacifique ${ }^{(39)} 》$.

Cette «arme pacifique», les Chœurs de fondeurs entendent l'utiliser pour "alerter» afin de prévenir de tels licenciements. Les anciens salariés de Metaleurop ont en effet une conscience très claire $\mathrm{du}$ caractère exceptionnel de la fermeture dont ils font l'objet en 2003 et mobilisent le terme de "référence» ou d' "exemple» de manière récurrente pour en parler mais aussi pour désigner la spécificité et la valeur de leur combat. C'est à

(36) Entretien recueilli par Judith Hayem le 14 novembre 2007 avec Michel, 53 ans, ancien agent de maîtrise, créateur et directeur de la Scop Aced.

(37) Dans le film, l'ouvrier qui s'exprime passe par les étapes successives décrites avant de se redresser et de créer sa Scop dans le but de décontaminer le site métallurgique.

(38) Entretien recueilli par Judith Hayem le 25 novembre 2007 avec Vladimir, 49 ans, ancien agent de maîtrise, en recherche d'emploi.

(39) Idem. 
ce titre qu'ils entendent faire "øuvre de péda$\operatorname{gogie}^{(40)}$ » en partageant leurs expériences, leurs acquis et leurs apprentissages et répondent aussi bien aux sollicitations des entreprises confrontées au même sort les années suivantes qu'aux invitations des syndicalistes de Coïmbra (Portugal). Ceux qui maîtrisent bien les dossiers prodiguent même des conseils extrêmement pointus sur les modalités d'intervention du comité d'entreprise, la façon de s'adresser à la presse, de guider les experts, de concevoir une association, etc. Il s'agit à chaque fois de disposer de nouveaux possibles et de suggérer que les salariés, victimes de licenciements, ont toute légitimité non seulement à les contester mais encore à se prononcer sur ces derniers et sur les suites à leur donner.

Par-delà la diversité des raisons évoquées, ce qui unit les interpellations créatives est la conviction de leurs promoteurs de ne pas parler en leur seul nom, quand bien même ils agissent de manière individuelle, mais de s'exprimer dans l'intérêt collectif, celui des salariés mobilisés et, plus largement, celui de la société dans son ensemble. Cette «subjectivation civique» (HAYEM, 2010) qui autorise, suscite et légitime la prise de parole et l'expression de chacun, même ceux qui n'en sont pas coutumiers comme les ouvriers, est le fruit de la mobilisation contre la fermeture et de l'inter-reconnaissance qu'elle a induite entre salariés. Celle-ci a donné à ceux qui avaient fait le choix de la lutte le sentiment d'appartenir à un collectif et d'y tenir chacun une place égale, ce qui les légitimait à se prononcer sur ce qui avait lieu, personnellement certes mais au nom de l'intérêt général. Ressource majeure de la mobilisation, «cette égalité universelle parlante» (RANCIÈRE, 1995) est cependant fragile car elle ne tient que tant que le principe d'égalité entre membres de l'association se maintient lui aussi. Or l'observation de l'association sur la longue durée révèle que cela n'a pas été le cas.

\section{Coups d'arrêt à l'interpellation créative}

Fin 2007, les interpellations créatives cessent. Au même moment, l'association connaît deux tournants notables dont je voudrais montrer ici l'articulation avec cette interruption.

Les tensions au sein de Chœurs de Fondeurs se font vives si bien qu'insensiblement, elle se divise en deux groupes opposés et rivaux, autrefois opposés par leur appartenance (ou leur proximité) syndicale respective à la CFE-CGC ou à la CFTC, et la CFDT. Désormais, la division est également structurée par des différences d'âge, entre les plus

(40) Idem. de 50 ans dispensés de recherche d'emploi et les plus jeunes en quête d'un autre emploi.

Ces deux groupes s'engagent à peu près au même moment dans des entreprises distinctes : création d'un projet de Centre du métal (Metallia), pour l'un; dépôt de ce qui reste des archives de Metaleurop aux Archives du monde du travail à Roubaix, pour l'autre.

En observant comment se mettent en place ces opérations, on comprend que l'égalité a fait long feu et que les rapports hiérarchiques sont de retour. L'orientation de ces nouvelles activités créatives vers la mémoire plutôt que vers le possible découle directement de ce changement marquant dans la subjectivité collective de l'association.

C'est l'autonomisation financière et physique de la fonction dite «d'accompagnement social» du reste des activités de l'association qui a suscité les premières tensions, bien que chacun reconnaisse le travail remarquable accompli par les deux anciens délégués syndicaux qui en ont pris la responsabilité. Dans ses travaux, Corteel (2009a, 2009b) analyse l'important exercice de «médiation» que ces derniers ont réalisé avec les institutions en faveur du retour à l'emploi des ex-salariés de Metaleurop et met en lumière l'importance et l'originalité de cette démarche. Ce n'est donc pas la qualité du travail qui pose problème mais sa rémunération et son indépendance. Alors que les membres de Chœurs de fondeurs sont des bénévoles(41), l'accompagnement social a été financé par des CDD que se sont partagés, sous le titre de "référents sociaux", deux anciens cadres commerciaux, Suzy et Didier, ex-délégués syndicaux de la CFE-CGC. S'ils n'ont pas ménagé leur peine souvent au-delà des heures rémunérées, cette différence de statut a fait grincer quelques dents, suscitant des remarques acerbes sur la façon dont certains ont su mieux tirer avantage de la situation que d'autres dans l'association (42). Il ne fait aucun doute par ailleurs que leurs anciennes fonctions les ont dotés de qualités et de compétences essentielles pour le travail à mener (MAZADE, 2010). Reste que certains y ont vu une forme d'instrumentalisation des objectifs de l'association en vue de retrouver / conserver un emploi. À y regarder de plus près, on constate que ce sont des personnes autrefois proches de la CFDT qui s'expriment de la sorte et il paraît évident qu'on retrouve là la trace de rivalités et de conceptions syndicales plus anciennes. Ce ressentiment latent s'accentue lorsque, après la fin de la cellule de reclassement pilotée par l'ANPE, la commission «accompagnement social» dont les deux intéressés ont la charge prend résidence non

(41) Les «référents sociaux», devenus «chargés de mission» pour leur projet de musée, sont toujours parvenus à faire financer par le Fonds social européen les postes qu'ils se sont créés. (42) Propos recueillis lors d'une discussion téléphonique en préparation d'une mission par Judith Hayem auprès de Richard, 60 ans, ancien ingénieur, dispensé de recherche d'emploi. 
pas dans les locaux de Chœurs de fondeurs, mis à sa disposition en mairie, mais dans un préfabriqué situé face à l'église du village, arguant que celui-ci offre plus de confidentialité. Ce local appartient à la Scop Aced (Au cœur de l'emploi durable) fondée par Michel, qui demeure par ailleurs membre de Chœurs de fondeurs. Exigu et lieu de passage incessant d'anciens salariés venus papoter, ce local n'est objectivement pas plus adapté aux entretiens confidentiels que la salle privée prévue en mairie, à l'écart du bureau des Chœurs de fondeurs. Mais en allant place de l'église on sait qu'on évitera de croiser «ceux de la mairie». Cette séparation est donc vécue comme l'incarnation d'une scission.

Or ce conflit ne parvient pas à se verbaliser et encore moins à se résoudre ouvertement au sein $\mathrm{du}$ conseil d'administration ou des assemblées générales. Et ce, d'autant moins que le président d'honneur et ancien chef de file de l'intersyndicale, Farid, qui a retrouvé un emploi d'agent de maîtrise mais dans le Sud de la France, n'assume plus désormais sa fonction de conciliateur et d'arbitre. C'est bien là une indication que quelque chose s'est rompu dans l'unité du collectif et quant à la liberté et l'égalité de parole. D'ailleurs, ceux de la place de l'église se mettent dans le même temps à critiquer les décisions de certains de leurs collègues du conseil d'administration, jugées «autoritaires et abusives (43) », et les rapprochent de ce qui se passait autrefois à l'usine.

Comme pour confirmer la division, les deux entités rivales ainsi constituées s'engagent dans deux projets patrimoniaux distincts rattachés au passé de Metaleurop ${ }^{(44)}$. Le groupe de la mairie, autour de Richard l'ingénieur, se lance dans le dépôt des archives de l'usine aux Archives du monde du travail (AMT) à Roubaix. L'opération est officiellement promue par Chœurs de fondeurs et montée avec l'aide de l'archiviste en chef des AMT. Ce projet est entièrement bénévole et vise notamment à inciter la recherche en histoire sociale sur cette usine. Dans ce groupe, les cadres n'ont souvent plus d'enjeux personnels d'emploi, étant préretraités ou

(43) Entretien recueilli par Judith Hayem le 29 novembre 2007 avec Suzy, 56 ans, Didier, 58 ans, et Alain, plus de 60 ans, respectivement anciens acheteurs et formateur. Suzy et Didier sont en CDD financés par le FSE comme chargés de mission pour le futur centre Metallia.

(44) La tension manifeste au sein de l'association et les propos que je recueillais à l'époque m'ont placée dans une situation difficile. Il me paraissait évident que seuls un dialogue et une confrontation franche entre les deux entités pourraient sauver l'association dont le CA connaissait alors une "épidémie de démissions» selon les termes de Pierre. Or susciter ce débat aurait signifié rompre l'anonymat des entretiens et outrepasser mon rôle de chercheuse en me faisant arbitre et en devenant militante au sein du collectif - ce que, du reste les statuts de l'association ne permettent pas légalement. Malgré ma sympathie politique pour Chœurs de fondeurs et mon amitié pour plusieurs acteurs des interpellations créatives, j'ai décidé de ne pas intervenir. dispensés de recherche d'emploi, eu égard à leur âge.

Le groupe de la place de l'église s'attelle quant à lui avec une grande discrétion à un projet de Centre du métal voué à parler du passé de l'usine mais surtout de l'avenir des métaux et de leur usage dans le monde. Le projet est porté par certains membres de Chœurs de fondeurs, triés sur le volet, et avec l'appui d'Aced. Pour le mener à bien, Suzy et Didier sont parvenus à obtenir un nouveau financement du FSE au titre de chargés d'étude. Ils espèrent s'employer durablement dans la structure si elle vient à exister et rêvent que la démarche attire de nombreux touristes et crée ainsi de nouveaux emplois dans la région. L'atmosphère de travail qui règne lors de la réalisation des deux projets manifeste symptomatiquement que l'égalité des statuts et l'égale capacité de penser qu'elle conférait à tous sont désormais des principes caducs. Les promoteurs du musée, Suzy, Didier et Alain m'expliquent qu'ils restent discrets car ils craignent de se voir "piquer» une bonne idée dans un environnement régional concurrentiel sur le tourisme industriel : Musée de la mine à Leuwarde, Louvre-Lens, Cockerie de Drocourt, etc. Surtout, ils développent en substance l'idée que "les autres» Chœurs de fondeurs ne sont pas "prêts à entendre la démarche globale» de ce tourisme industriel, ils sont "restés sur la mémoire de Metaleurop Malfidano»; "Eux, ne sont pas concrets, on leur présente [1e projet] mais eux n'ont pas, je crois, pris conscience.» Ces extraits d'entretiens ${ }^{(45)}$ ne sont que quelques exemples parmi d'autres de la division statutaire qui se réinstalle: «nous » sommes capables de mener à bien le projet de Centre du métal et de l'animer, «eux» ne le sont pas, argumentent mes interlocuteurs. Les Chœurs de fondeurs autrefois ouvriers ou agents de maîtrise ne s'y trompent pas, et moins encore ceux qui ont pratiqué l'interpellation créative. Louis, sollicité par les promoteurs du Centre du métal, en raison de ses talents de photographe, m'explique :

"Dans le musée, t'as ceux qui gèrent et t'as ceux qui trient. Donc être dans le groupe, je le fais pas. Je me suis retiré. [...] C'est la mentalité Metaleurop. T'as les gens qui sont au-dessus et t'as les gens qui sont en bas. Bon moi, j'étais que l'ouvrier, j'étais au niveau le plus bas quoi. "»

(Entretien recueilli par Judith Hayem le 15 novembre 2007 avec Louis, 56 ans, ancien ouvrier conducteur de locomotive sur le site, dispensé de recherche d'emploi. Louis rejoindra finalement le projet de Centre du métal quand il sera avéré que les interpellations créatives n'auront plus lieu. Il n'a pas non plus participé au dépôt des archives aux AMT.)

(45) Tous ces extraits sont tirés de l'entretien recueilli par Judith Hayem le 29 novembre 2007 avec Suzy, 56 ans, Didier, 58 ans et Alain plus de 60 ans, respectivement anciens acheteurs et formateur. 
À la même période, certains participants au projet d'archivage dénoncent un phénomène du même ordre en parlant du comportement de Richard, pilier de l'association, ancien ingénieur à l'usine, à l'égard de ses anciens subordonnés. Vladimir s'emporte ainsi :

"Il a gardé l'usine dans la tête, il se comporte comme un chef, il se croit à l'usine, il a oublié qu'après le licenciement, on était tous pareils!»

(Entretien recueilli par Judith Hayem le 14 novembre 2007 avec Vladimir, 49 ans, ancien agent de maitrise, en recherche d'emploi.)

À entendre la description que Robert, un de ses responsables me fait du travail d'archivage, on pourrait d'ailleurs croire qu'il décrit un protocole opérationnel ayant trait au fonctionnement d'un département de l'usine : ce sont les mêmes mots, les mêmes tournures, les mêmes procédures aussi :

"On a fixé un ordre de travail en se fixant sur l'organigramme de l'usine, l'organigramme qualité, en disant on va récupérer des documents là, là, là. [...] On a choisi un certain nombre de personnes [...] agréées par [le leader du projet] et l'ensemble des chefs d'équipe [...] sachant qu'il fallait des gens sérieux, honnêtes et confidentiels [...] parce que les archives, c'est confidentiel [...].»

(Entretien recueilli par Judith Hayem le 30 novembre 2007 avec Robert, 61 ans, directeur de l'atelier raffinage zinc, retraité.)

La logique choisie a du sens pour un travail d'archivage mais l'écho avec les processus du temps de l'activité de l'usine est manifeste et troublant. Loin du «tous pareils», les procédés hiérarchiques et statutaires sont réinstaurés et avec eux cesse la possibilité des interpellations créatives. Que Chœurs de fondeurs se consacre à la mise en mémoire et en histoire ${ }^{(46)}$ - qui n'était pas, elle non plus, dans les statuts de l'association - participe d'une manière renouvelée à la manifestation positive du travail de l'usine (Gimel, 2009). Il s'agit là encore de laisser des traces et une forme de «vérité» sur ce qui était fait à Metaleurop, son importance, son intérêt. Cependant, cette focalisation nouvelle ${ }^{(47)}$ représente une forme de clôture sur le passé, qui semble désormais révolu. En cela, elle se distingue nettement de la création du dissensus qui offrait, y compris à propos de faits passés, la capacité de mettre en présence des politiques différentes: logique des

(46) L'exposition Fondre en chœurs, initiative de l'association en 2006, a été un précédent dans le registre mémoriel.

(47) La mise en valeur du patrimoine industriel répond ici, comme dans d'autres régions, à la demande des institutions politiques et connaît de nombreux antécédents dans le Nord de la France; c'est aussi une manière de donner une attractivité touristique à un territoire qui se désindustrialise et d'éteindre le militantisme de l'association. Voir par exemple les analyses de Laurent BAZIN, sur Metallia (2014, à paraître) et l'épilogue de mon rapport de recherche (HAYEM, 2010) qui décrit, à la veille des élections régionales, la présentation du projet par Aced en présence de tous les élus de la région. salariés, logique des institutions, logique de l'écologie, logique des actionnaires, etc., chacune ouvrant vers différents possibles advenus ou non advenus, et offrant donc la possibilité de lutter contre la fermeture de Metaleurop et partant, de lutter contre d'autres fermetures similaires.

À l'heure actuelle, les archives de Metaleurop sont consultables à Roubaix. Aced compte plus de «100 membres dont $95 \%$ d'anciens métallos $(48) »$. Metallia, le Centre du métal, reste de proportions modestes, et n'a pas les ressources nécessaires pour embaucher ses fondateurs en CDD, mais organise annuellement des manifestations «pour conserver la mémoire de Metaleurop et parler de l'avenir de l'acier ${ }^{(49)} \gg$. La scission de 2007 n'a entamé ni les effectifs ni la poursuite des actions juridiques en cours mais le fonctionnement associatif semble être entré dans une forme de routinisation et sa polarisation vers les actions mémorielles s'accentue. distinctes ${ }^{(50)}$ : la phase défensive du combat contre la fermeture (de janvier à avril 2003) qui a mobilisé un répertoire d'action classique; la poursuite $\mathrm{du}$ «tous ensemble» au sein de Chœurs de fondeurs avec le travail de cinq commissions et la pratique originale des interpellations créatives (d'avril 2003 à avril 2007); puis, après 2007, la division de l'association en deux entités, sans dissolution de celle-ci, mais avec le retour d'un fonctionnement hiérarchisé et une inflexion de ses activités vers la patrimonialisation.

Qu'est-ce qui dès lors distingue l'action de Chœurs de fondeurs d'expériences anciennes ou plus récentes dans lesquelles des salariés font appel aux ressorts de la création? Portées par quelquesuns, sans anticipation initiale, les interpellations créatives ont été légitimées par l'association qui les a soigneusement répertoriées dans l'Écrit du Chœur et leur a attribué un budget de fonctionnement; mais elles n'ont pas fait l'objet d'une stratégie préalablement définie comme lors de la création de la Radio Lorraine Cour d'acier initiée par la CGT au début des années 1980, qui envoyait des journalistes

(48) Propos du vice-président de l'association recueillis par la télévision de l'agglomération de Hénin-Carvin dans le reportage «Gros plan sur Aced Metallia»; disponible en ligne à l'adresse : http://www.iltv.fr/Services-en-ligne/Espace-documentaire/ Videos/LE-JOURNAL-2013/2013_03_06_journal/Gros-plansur-ACED-Metallia; consulté le 5 juin $201 \overline{3}$.

(49) Idem.

(50) Pour apprécier dans quelle phase nous sommes aujourd'hui, un retour sur le terrain serait nécessaire. L'examen du nouveau site des CDF m'incite à penser que nous sommes toujours dans la phase 3 : www.choeursdefondeurs.fr/, consulté le 5 juin 2013. 
parisiens à Longwy par exemple (HAYES, 2013). En outre, même quand l'interpellation créative s'appuie sur le travail des professionnels de la culture ou en appelle aux «intellectuels», il ne s'agit pas pour autant de déléguer à d'autres le soin de parler pour soi ou de solliciter une prise de parti de la part de quelqu'un d'éminent mais d'utiliser la puissance de diffusion de ces «porteurs de paroles». Projections, débats, lectures, expositions ou créations personnelles sont donc autant d'occasions saisies pour faire connaître la pensée des Chœurs de fondeurs et proposer d'autres possibles après que le travail initial mené en direction des journalistes et des réalisateurs a permis de poser dans le débat public la question de la responsabilité sociale des entreprises, tant en termes de délocalisation que de pollution industrielle, et surtout sans que les ouvriers soient oubliés ou désignés comme coupables de ce qui leur arrive. Grâce aux questions posées, aux recommandations formulées et aux débats ouverts, ces interpellations ont contribué, au moins localement, à l'ouverture de «scènes politiques nouvelles». Et ce, au point que l'évocation du conflit à chaque occasion possible soit parfois saluée par les politiques locaux d'un soupir éloquent: "Encore les Chours de fondeurs ${ }^{(51) !}$ !.."

Avec les interpellations politiques, auxquelles se sont joints le combat juridique et la volonté de participer à la revitalisation du territoire, les Chœurs de fondeurs ont permis que la question d'une législation contre les délocalisations soit aujourd'hui débattue de façon contradictoire. De ce point de vue, la «médiatisation à l'envers » constitue une manière inventive et efficace de prendre la mesure des enjeux contemporains de l'accès à et surtout du contenu de l'information, tout en restaurant une place pour la parole ouvrière. En dépassant le témoignage, la mise en mémoire, et l'important enjeu de reconstruction de soi et en donnant une ambition préventive et prescriptive à ses pratiques culturelles et créatives, Chœurs de fondeurs se distingue d'autres associations de salariés qui, dans les années 2000, ont, elles aussi, fait de l'expérience de l'usine et de la fermeture des créations artistiques ${ }^{(52)}$. Dans une séquence politique où la représentativité politique et syndicale des ouvriers est affaiblie, où le mot «ouvrier» luimême est quasi absent du débat public, les porteurs d'interpellations créatives se présentent comme des membres de la «classe ouvrière»-parfois en dépit de leur statut social réel - pour prendre la parole au nom du collectif et pour prescrire l'avenir plus encore que conserver le passé.

Cependant, cette pratique inventive ne parvient pas à se stabiliser dans la longue durée. Passés l'émulation et l'enthousiasme des luttes, le repli sur les identités professionnelles et les anciennes rivalités entre syndicalistes, mais aussi les enjeux de création d'activité professionnelle pour certains, brisent la notion revisitée de «classe ouvrière» et la «subjectivité civique» qui avaient émergé. Alors que se cherche, avec les interpellations créatives, un nouveau type d'organisation et d'intervention politique, Chœurs de fondeurs, association loi 1901 originale et interlocutrice efficace des institutions, peine à discuter de son organisation interne et de l'évolution de ses objectifs. Pour que perdure une subjectivité collective unifiée, capable d'affirmer au présent l'existence des ouvriers, il a probablement manqué à l'association, et en particulier aux porteurs d'interpellations, la possibilité de débattre collectivement des principes dont ils se dotaient et de l'idée qu'ils se faisaient du collectif qu'ils formaient.

\section{Bibliographie}

Bergeron A., Doray B. (2005), «Les “privés d'emploi” : la culture pour miroir», Ethnologie française, vol. 35, $\mathrm{n}^{\circ} 4$, pp. 643-652.

Balasinski J., Mathieu L. (2006), Art et contestation, Rennes, Presses universitaires de Rennes.

BAZIN L. (2014), «Anthropologie, patrimoine industriel et mémoire ouvrière. Vers une recontextualisation critique», L’homme et la société, $\mathrm{n}^{\circ} 192$, à paraître.

Bon F. (2004), Daewoo, Paris, Fayard.

Bourdieu P. (1982), Ce que parler veut dire : l'économie des échanges linguistiques, Paris, Fayard.

(51) Entretien recueilli par Judith Hayem le 29 novembre 2007 avec Suzy, 56 ans, Didier, 58 ans et Alain, plus de 60 ans, respectivement anciens acheteurs et formateur.
Corteel D. (2009a), «Lutter pour la reconversion d'un site industriel et la dignité des hommes qui l'ont fait vivre. Analyse d'expériences menées par les ex-salariés de Metaleurop Nord», in Causer J.-Y., Durand J.-P., Gasparini W. (coord.), Les identités au travail. Analyses et controverses, Toulouse, Octarès, pp. 113-122.

CorTeEL D. (2009b), «Fermetures d'usine : les associations d'anciens salariés comme espaces de médiation originaux », Formation emploi, n ${ }^{\circ} 108$, pp. 53-65.

Corteel D., Duhin A., Hayem J. (2009), « Metaleurop : "dal defensive al creativo". Cronaca e analisi di una lotta singolare», in Romittelli V., Fuori dalla società della conoscenza, Roma, Infinito, pp. 265-280.

(52) Par exemple et de manière non exhaustive Levi's, Daewoo, Cellatex, Mosley, Mollex (MArtin, 2001; Bon, 2004; BERGERON, Doray, 2005). 
FAJARDIE F. (2003), Metaleurop : paroles ouvrières, Paris, Mille et une nuits.

Fermetures de boîtes... et après ? (2007), Actes des rencontres à la Maison des hommes et des techniques à Nantes, mars 2005, Maison des hommes et des techniques, Édition du Centre d'histoire du travail, Nantes (livre + cédérom).

GAulin M.-J. (2005), Metaleurop : une crise locale aux causes internationales et aux conséquences nationales, Mémoire de maîtrise sous la direction de Robert Franck, université Paris-I.

Gimel J. (2009), Mémoire ouvrière, mémoires d'ouvriers. Une analyse sociologique des pratiques mémorielles des ouvriers de l'usine Metaleurop, Mémoire de maîtrise de sociologie sous la direction d'Alain Chenu, Institut d'études politiques de Paris.

Hayem J. (2010), «La mobilisation créative des ex-ouvriers de Metaleurop Nord», Rapport de recherche remis au PUCA en 2011 dans le cadre de la recherche sur La citoyenneté urbaine : formes d'engagement et enjeux de solidarité dirigée par Bernard Eme, pp. 230-304.

Hayes I., (2013), «Les limites d'une médiation militante. L'expérience de Radio Lorraine Cœur d'acier, Longwy, 1979-1980», Actes de la recherche en sciences sociales, n ${ }^{\circ}$ 196-197, pp. 84-101.

LAZARUS S. (2001), «Anthropologie ouvrière et enquêtes d'usine : état des lieux et problématique», Ethnologie française, vol. 31, $\mathrm{n}^{\circ} 3$, pp. 389-400.

Lazarus S. (1996), Anthropologie du nom, Paris, Seuil.

Linhart D., Rist B., Durand E. (2002), Perte d'emploi, perte de soi, Paris, Erès.

Lomba C. (2013), «Restructurations industrielles: appropriations et expropriations des savoirs ouvriers ", Actes de la recherche en sciences sociales, $n^{\circ} 196-197$, pp. 34-53.

Martin C. (2001), Les mains bleues, Lille, Éditions du Sansonnet.

Mariette A. (2005), “"Silence, on ferme !”», Ethnologie française, vol. 35, $\mathrm{n}^{\circ} 4$, pp. 653-666.

Mathieu L. (2009), Les années 70, un âge d'or des luttes?, Paris, Textuel.

Mazade O. (2005), «Cellules de reclassement et individualisation du traitement du chômage. Le cas de
Metaleurop et des Houillères du Nord. », Revue de l'Ires, $\mathrm{n}^{\circ} 47$, pp. 195-214.

Mazade O. (2006), Le reclassement des ex-salariés de Metaleurop Nord: enquête quantitative, rapport de recherche, Clersé, mars.

MAZAde O. (2010), La reconversion des hommes et des territoires. Le cas Metaleurop, Paris, L'Harmattan, coll. «Logiques sociales».

MAzAdE O. (2013a), «L'affaire Metaleurop. Une dénonciation impossible ?», Terrains et travaux, $\mathrm{n}^{\circ} 22$, pp. 23-38.

MAzade O. (2013b), “"Patron voyou” : de la désignation publique à la sanction juridique. L'affaire Metaleurop»", Champ pénal/Penal Field [En ligne], vol. X; disponible à l'adresse : http://champpenal.revues.org/8431; consulté le 24 mars 2014.

Mouchard D. (2009), «L'intellectuel spécifique», in Filleule O., Mathieu L., Pechu C. (dir.), Dictionnaire des mouvements sociaux, Paris, Presses de Sciences Po, pp. 307-312.

RANCIÈRE J. (2000), Le partage du sensible. Esthétique et politique, Paris, La Fabrique.

RANCIÈRE J. (1995), La mésentente, Paris, Galilée.

RANCIÈRe J. (2005), La haine de la démocratie, Paris, La Fabrique.

RANCIÈRe J. (2007), Le spectateur émancipé, Paris, La Fabrique.

Ross K. (2005), Mai 68 et ses vies ultérieures, Bruxelles, Complexe-Le Monde diplomatique.

Sinigaglia J. (2012), Artistes, intermittents, précaires en lutte. Retour sur une mobilisation paradoxale, Nancy, Presses universitaires de Nancy.

Surubaru A. (2010), «De la sous-traitance à la délocalisation. Les restructurations chez DIM», in Jobert A., Didry C. (dir.), L'entreprise en restructuration. Dynamiques institutionnelles et mobilisations collectives, Rennes, Presses universitaires de Rennes, pp. 235-248.

Zuindeau B., Letombe G., Masson E. (2010), «Risques de pollution et action collective dans le bassin minier (cas de Metaleurop) : défection ou prise de parole ?», in Petit O., Herbert V. (coord.), Risque environnemental et action collective : Application aux risques industriels et d'érosion côtière dans le Pas-de-Calais, Paris, Lavoisier, coll. «Sciences du risque et du danger», pp. 79-93. 


\section{Annexe}

Tableau : Le noyau des adhérents actifs de l'association Chœurs de fondeurs (CDF) ${ }^{(53)}$

\begin{tabular}{|c|c|c|c|c|c|c|}
\hline Interviewé & $\begin{array}{l}\text { Situation } \\
\text { familiale }\end{array}$ & $\begin{array}{l}\text { Fonction en } 2003 \text { et } \\
\text { éléments connus du } \\
\text { parcours professionnel } \\
\text { antérieur }\end{array}$ & $\begin{array}{l}\text { Âge } \\
\text { en } \\
2003\end{array}$ & $\begin{array}{c}\text { Appartenance } \\
\text { syndicale et autres } \\
\text { engagements } \\
\text { militants }\end{array}$ & $\begin{array}{l}\text { Investissements } \\
\text { créatifs }\end{array}$ & $\begin{array}{c}\text { Position dans } \\
\text { l'association, activité } \\
\text { (bénévole ou salariée), } \\
\text { situation professionnelle } \\
\text { en } 2007\end{array}$ \\
\hline Pierre & $\begin{array}{l}\text { Marié, } 3 \\
\text { enfants }\end{array}$ & $\begin{array}{l}\text { Ingénieur, a travaillé en } \\
\text { Afrique, Allemagne, Italie. }\end{array}$ & 57 & $\begin{array}{l}\text { Non syndiqué, } \\
\text { étudiant militant en } \\
1968 .\end{array}$ & $\begin{array}{l}\text { Historien amateur; } \\
\text { nombreuses } \\
\text { interventions lors } \\
\text { des projections et } \\
\text { expositions, travail } \\
\text { pour les archives. }\end{array}$ & $\begin{array}{l}\text { Membre régulier du conseil } \\
\text { d'administration (CA) à } \\
\text { divers postes, bénévole. } \\
\text { Chômeur dispensé de } \\
\text { recherche d'emploi. }\end{array}$ \\
\hline Louis & $\begin{array}{l}\text { Marié, } 2 \\
\text { enfants }\end{array}$ & $\begin{array}{l}\text { Conducteur de train sur } \\
\text { le site. Ouvrier agricole à } \\
\text { l'adolescence puis travaille } \\
\text { dans le bâtiment quelques } \\
\text { années avant d'être } \\
\text { embauché à Metaleurop. }\end{array}$ & 52 & / & $\begin{array}{l}\text { Photos de la } \\
\text { fermeture et de la } \\
\text { destruction du site } \\
\text { et de toutes les } \\
\text { actions militantes } \\
\text { et autres de CDF ; } \\
\text { nombreuses } \\
\text { interventions lors } \\
\text { des projections } \\
\text { et expositions, } \\
\text { participe au projet } \\
\text { du Centre du métal, } \\
\text { Metallia. }\end{array}$ & $\begin{array}{l}\text { Bénévole, très actif mais } \\
\text { sans responsabilité au sein } \\
\text { du CA. } \\
\text { Chômeur dispensé de } \\
\text { recherche d'emploi (rachat } \\
\text { de ses trimestres en tant } \\
\text { qu'ouvrier agricole). }\end{array}$ \\
\hline Vladimir & $\begin{array}{l}\text { Marié, } 2 \\
\text { enfants }\end{array}$ & $\begin{array}{l}\text { Agent de maîtrise (parcours } \\
\text { professionnel non } \\
\text { reconstitué). }\end{array}$ & 45 & Non syndiqué. & $\begin{array}{l}\text { Écriture ; } \\
\text { nombreuses } \\
\text { interventions lors } \\
\text { des projections et } \\
\text { expositions. }\end{array}$ & $\begin{array}{l}\text { Bénévole, a été membre du } \\
\text { CA, démissionne en } 2007 . \\
\text { Après un CDD au sein de la } \\
\text { Scop (société coopérative } \\
\text { et participative) de Michel } \\
\text { et en dépit de multiples } \\
\text { démarches et projets, } \\
\text { toujours au chômage en } \\
2007 .\end{array}$ \\
\hline Thimotée & $\begin{array}{l}\text { Marié, } 2 \\
\text { enfants }\end{array}$ & $\begin{array}{l}\text { Chef de poste. Embauché } \\
\text { comme intérimaire à } \\
\text { Metaleurop à partir de } 1980 \\
\text { puis électricien passe de } \\
\text { OP1 (ouvrier qualifié de } \\
\text { niveau 1) à OP3 (ouvrier } \\
\text { qualifié de niveau 3) puis } \\
\text { chef de poste en 2003. }\end{array}$ & 45 & $\begin{array}{l}\text { A été un temps } \\
\text { délégué syndical } \\
\text { FO avant } 2003, \\
\text { «famille PS », } \\
\text { militant de la } \\
\text { Confédération } \\
\text { nationale du } \\
\text { logement. }\end{array}$ & $\begin{array}{l}\text { A composé et } \\
\text { enregistré un album } \\
\text { relatif à la lutte } \\
\text { (« Métallo ») avec } \\
\text { son groupe semi- } \\
\text { professionnel. Se } \\
\text { produit en concert } \\
\text { pendant la séquence } \\
\text { de lutte. }\end{array}$ & $\begin{array}{l}\text { Membre bénévole de } \\
\text { l'association. } \\
\text { En } 2007 \text { travaille dans } \\
\text { l'automobile. }\end{array}$ \\
\hline Michel & $\begin{array}{l}\text { Marié, } 6 \\
\text { enfants }\end{array}$ & $\begin{array}{l}\text { Agent de maîtrise en } \\
2003 \text {. A travaillé } 5 \text { ans } \\
\text { dans les Houilles puis } \\
\text { débute comme balayeur } \\
\text { à Metaleurop en } 1975 \text {, } \\
\text { formation en physique } \\
\text { (susceptible de valoir une } \\
\text { équivalence en Master } 2 \\
\text { par validation des acquis de } \\
\text { l'expérience), gravit tous } \\
\text { les postes jusqu'à devenir } \\
\text { agent de maîtrise en } 2003 \text {. }\end{array}$ & 49 & $\begin{array}{l}\text { Fils d'ouvrier } \\
\text { textile, petit fils } \\
\text { de mineur. A été } \\
\text { délégué syndical } \\
\text { CGT mais « a } \\
\text { claqué la porte » en } \\
\text { 1978-1979, reste } \\
\text { syndiqué et proche } \\
\text { des idées de la } \\
\text { CGT. }\end{array}$ & $\begin{array}{l}\text { Protagoniste } \\
\text { de Metaleurop, } \\
\text { Germinal 2003, } \\
\text { nombreuses } \\
\text { interventions lors } \\
\text { des projections } \\
\text { et expositions, } \\
\text { fondateur de la } \\
\text { Scop Aced (Au } \\
\text { cœur de l'emploi } \\
\text { durable), projet de } \\
\text { Centre du métal, } \\
\text { Metallia. }\end{array}$ & $\begin{array}{l}\text { Membre bénévole de } \\
\text { l'association. } \\
\text { A fondé une Scop de } \\
\text { dépollution du site après } \\
\text { la fermeture puis plusieurs } \\
\text { autres entreprises associées } \\
\text { (laverie, démantèlement } \\
\text { d'ordinateurs), dont } \\
\text { certaines ferment ensuite. } \\
\text { En } 2007 \text { il emploie encore } \\
\text { plusieurs personnes et } \\
\text { parvient à en vivre. }\end{array}$ \\
\hline
\end{tabular}

(53) Sont présentées ici les principales caractéristiques des personnes interviewées pendant l'enquête. Celle-ci n'ayant pas pris la forme d'entretiens biographiques, certaines informations concernant le parcours des interviewés ne sont pas disponibles. Les informations présentées correspondent à ce que l'interviewé a souhaité mettre en avant de son parcours pour expliquer ses engagements au moment de l'entretien. 


\begin{tabular}{|c|c|c|c|c|c|c|}
\hline Interviewé & $\begin{array}{l}\text { Situation } \\
\text { familiale }\end{array}$ & $\begin{array}{l}\text { Fonction en } 2003 \text { et } \\
\text { éléments connus du } \\
\text { parcours professionnel } \\
\text { antérieur }\end{array}$ & $\begin{array}{c}\text { Âge } \\
\text { en } \\
2003\end{array}$ & $\begin{array}{c}\text { Appartenance } \\
\text { syndicale et autres } \\
\text { engagements } \\
\text { militants }\end{array}$ & $\begin{array}{l}\text { Investissements } \\
\text { créatifs }\end{array}$ & $\begin{array}{c}\text { Position dans } \\
\text { l'association, activité } \\
\text { (bénévole ou salariée), } \\
\text { situation professionnelle } \\
\text { en } 2007\end{array}$ \\
\hline Alain & / & $\begin{array}{l}\text { Engagé à Metaleurop dans } \\
\text { le secteur chimie puis muté } \\
\text { à la formation de } 1979 \text { à } \\
1999 \text { puis service sécurité } \\
\text { de } 1999 \text { à } 2003 \text {. Retraite } \\
\text { anticipée en } 2003 \text {. }\end{array}$ & +55 & $\begin{array}{l}\text { Famille } \\
\text { d'agriculteurs. } \\
\text { Information } \\
\text { sur l'affiliation } \\
\text { syndicale non } \\
\text { disponible. }\end{array}$ & $\begin{array}{l}\text { Vidéaste amateur, } \\
\text { réalisation de } \\
\text { plusieurs films } \\
\text { sur l'usine et de } \\
\text { tous les montages } \\
\text { vidéos pour les } \\
\text { AG, collaboration } \\
\text { au film d'Attac, } \\
\text { a créé le logo des } \\
\text { fondeurs, initiateur } \\
\text { du projet de Centre } \\
\text { du métal, Metallia. }\end{array}$ & $\begin{array}{l}\text { Membre de l'association } \\
\text { bénévole. } \\
\text { Dispensé de recherche } \\
\text { d'emploi. }\end{array}$ \\
\hline Suzy & $\begin{array}{l}\text { Mariée, } 1 \\
\text { enfant }\end{array}$ & $\begin{array}{l}\text { Travaille enfant chez } \\
\text { Penarroya pendant les } \\
\text { grandes vacances, entre à } \\
\text { Metaleurop en } 1971 \text { aux } \\
\text { achats, puis promotions } \\
\text { internes. Acheteuse, statut } \\
\text { cadre. }\end{array}$ & 52 & $\begin{array}{l}\text { Déléguée syndicale } \\
\text { CGC, élue au CE. }\end{array}$ & $\begin{array}{l}\text { Initiatrice du projet } \\
\text { de Centre du métal, } \\
\text { Metallia. }\end{array}$ & $\begin{array}{l}\text { Membre du CA. } \\
\text { Salariée en CDD par le } \\
\text { FSE pour le suivi social de } \\
\text { la cellule de reclassement } \\
\text { de l'ANPE de } 2003 \text { à } 2007 \\
\text { puis salariée en CDD par } \\
\text { le FSE en tant que chargée } \\
\text { d'études sur le Centre du } \\
\text { métal à partir de } 2009 \text {. }\end{array}$ \\
\hline Didier & $\begin{array}{l}\text { Marié, } 1 \\
\text { enfant }\end{array}$ & $\begin{array}{l}\text { Entre à Metaleurop en } \\
1979 \text { comme responsable } \\
\text { de gestion puis commercial } \\
\text { et achats. Acheteur, statut } \\
\text { cadre. }\end{array}$ & 54 & $\begin{array}{l}\text { Délégué syndical } \\
\text { CGC, élu aux } \\
\text { prud'hommes. }\end{array}$ & $\begin{array}{l}\text { Initiateur du projet } \\
\text { de Centre du métal, } \\
\text { Metallia. }\end{array}$ & $\begin{array}{l}\text { Membre du CA. } \\
\text { Salarié en CDD par le FSE } \\
\text { pour le suivi social de la } \\
\text { cellule de reclassement de } \\
\text { l'ANPE de } 2003 \text { à } 2007 \\
\text { puis salarié en CDD par le } \\
\text { FSE en tant que chargée } \\
\text { d'études sur le Centre du } \\
\text { métal à partir de } 2009 \text {. }\end{array}$ \\
\hline Richard & $\begin{array}{l}\text { Marié, } 3 \\
\text { enfants }\end{array}$ & $\begin{array}{l}\text { Ingénieur (parcours } \\
\text { professionnel non } \\
\text { reconstitué). }\end{array}$ & 57 & $\begin{array}{l}\text { Famille membre } \\
\text { de la Jeunesse } \\
\text { agricole catholique } \\
\text { (JAC), délégué } \\
\text { CFTC élu au CE } \\
\text { (sympathisant } \\
\text { CFDT), engagé } \\
\text { contre le FN à } \\
\text { Hénin-Beaumont, } \\
\text { fondateur d'une } \\
\text { association d'aide } \\
\text { aux handicapés, } \\
\text { proche du } \\
\text { Mouvement } \\
\text { national des } \\
\text { chômeurs et } \\
\text { précaires (MNCP). }\end{array}$ & $\begin{array}{l}\text { Dépôt d'archives } \\
\text { aux Archives du } \\
\text { monde du travail à } \\
\text { Roubaix. }\end{array}$ & $\begin{array}{l}\text { Membre bénévole du CA, } \\
\text { porteur de l'action en } \\
\text { justice et de la réactivation } \\
\text { du bassin d'emploi. } \\
\text { Chômeur dispensé de } \\
\text { recherche d'emploi. }\end{array}$ \\
\hline Marie & $\begin{array}{l}\text { Mariée, } 1 \\
\text { enfant }\end{array}$ & $\begin{array}{l}\text { Secrétaire de direction, } \\
\text { assistante du directeur } \\
\text { technique. Statut cadre, } \\
\text { mais se considère comme } \\
\text { " ouvrière ». }\end{array}$ & 51 & Non syndiquée. & $\begin{array}{l}\text { Dépôt d'archives } \\
\text { aux Archives du } \\
\text { monde du travail à } \\
\text { Roubaix. }\end{array}$ & $\begin{array}{l}\text { Membre bénévole. } \\
\text { En 2007, CDD de quelques } \\
\text { mois comme secrétaire } \\
\text { pour Chœurs de fondeurs } \\
\text { dans le cadre du projet de } \\
\text { dépôt des archives aux } \\
\text { Archives du monde du } \\
\text { travail à Roubaix. }\end{array}$ \\
\hline Robert & / & $\begin{array}{l}\text { Agent de maîtrise } \\
\text { dans la chaussure puis } \\
\text { entré à Metaleurop en } \\
1979 \text { comme OS1 puis } \\
\text { adjoint aux expéditions, } \\
\text { contremaître et chef } \\
\text { d'atelier. En } 2003 \text { participe } \\
\text { au cabinet de reclassement. }\end{array}$ & 57 & $\begin{array}{l}\text { CFTC, partenaire } \\
\text { de Richard dans } \\
\text { l'association d'aide } \\
\text { aux handicapés. }\end{array}$ & $\begin{array}{l}\text { Dépôt d'archives } \\
\text { aux Archives du } \\
\text { monde du travail à } \\
\text { Roubaix. }\end{array}$ & $\begin{array}{l}\text { Membre bénévole. } \\
\text { En } 2007 \text { préretraité } \\
\text { dispensé de recherche } \\
\text { d'emploi. }\end{array}$ \\
\hline
\end{tabular}

